\title{
Prognostic value of tumor-associated macrophages in pancreatic cancer: a meta-analysis
}

This article was published in the following Dove Press journal:

Cancer Management and Research

\author{
Min $\mathrm{Yu}^{\mathrm{I}} *$ \\ Renguo Guan',* \\ Weifeng Hong ${ }^{2}$ \\ Yu Zhou' \\ Ye Lin' \\ Haosheng Jin' \\ Baohua Hou' \\ Zhixiang Jian' \\ 'Department of General Surgery, \\ Guangdong Provincial People's Hospital, \\ Guangdong Academy of Medical \\ Sciences, Guangzhou, Guangdong \\ Province, People's Republic of China; \\ ${ }^{2}$ Department of Medical Imaging, The \\ First Affiliated Hospital of Guangdong \\ Pharmaceutical University, Guangzhou, \\ People's Republic of China \\ *These authors contributed equally to \\ this work
}

\begin{abstract}
Objective: Tumor-associated macrophages (TAMs) deserve more focus because of its pivotal role in the development of solid tumors and they are related to poor outcomes of several tumors. However, the prognostic value of tumor-infiltrating TAMs in pancreatic cancer is still controversial.

Experimental design: We conduct a meta-analysis of 1699 patients in 13 studies by reviewing the studies in which the authors evaluated the prognostic value of TAMs density in pancreatic cancer. These studies were searched from inception to November 2018. Hazard ratios (HR) with their corresponding 95\% confidence intervals (95\% CIs) were assessed to explore the prognostic significance of TAMs in pancreatic cancer. Besides, we also conducted a subgroup analysis and sensitivity analysis. Two reviewers independently abstracted data.

Results: A total of 13 studies with 1699 patients were pooled in the analysis to evaluate prognostic value of TAMs in pancreatic cancer. The pooled HRs demonstrated CD68+ TAMs correlated with worse overall survival (OS) in pancreatic cancer patients $(\mathrm{HR}=1.41,95 \% \mathrm{CI}=1.05-1.90$, random effects model, $\mathrm{I}^{2}=82.5 \%, P=0.021$ ). And high generalized M2-TAMs density was significantly associated with poor $\mathrm{OS}\left(\mathrm{HR}=1.95,95 \% \mathrm{CI}=1.63-2.33\right.$, random effects model, $\mathrm{I}^{2}=59.2 \%$, $P=0.000)$ and disease-free survival $(\mathrm{HR}=1.83,95 \% \mathrm{CI}=1.43-2.36$, fixed effects model, $\left.\mathrm{I}^{2}=0.00 \%, P=0.000\right)$. Pooled analysis showed no significant correlation between elevated TAMs infiltration and lymph node metastasis, tumor stage, histological grade, sex, or tumor location.

Conclusion: The density of TAMs has an impact on the overall survival of pancreatic cancer patients. M2-TAMs can be recognized as a prognostic indicator in pancreatic cancer, which may serve as a potential therapeutic target in the treatment of pancreatic cancer.
\end{abstract}

Keywords: prognostic markers, survival, pancreatic cancer, macrophage, systematic review, meta-analysis

\section{Introduction}

Due to its invasive and metastatic characteristics, pancreatic ductal adenocarcinoma (PDAC), also known as pancreatic cancer, is characterized by one of the most malignant tumors. ${ }^{1}$ The difficulty in the early diagnosis makes matters worse. Even though there are many kinds of treatments for pancreatic cancer such as operative treatment, chemotherapy, and so on, patients who had pancreatic cancer still have a poor prognosis. Pancreatic cancer accounts for the seventh cause of cancer-related mortality in China. ${ }^{2}$ As a result, there is a need to identify more effective prognostic indicators. Without such enormous progress in the genomics and proteomics in the pancreatic cancers, numerous searchers change their targets to micro-environment of tumors, which are involved with heterogeneous inflammatory cells. These inflammatory cells contribute to the initiation, growth
Correspondence: Min Yu; Zhixiang Jian Department of General Surgery, Guangdong Provincial People's Hospital, Guangdong Academy of Medical Sciences, No. 106, Zhongshan Second Road, Guangzhou, Guangdong Province, People's Republic of China

Tel +86 I85 $8852 \quad$ II 68

Email yuminzhongda@163.com; jzx_118@I63.com 
and distant metastasis. ${ }^{3}$ Among them, tumor-associated macrophages are of great significance, originating from circulating monocytes. It is shown that tumor-associated macrophages can be divided into two classes of type 1 macrophages (M1) and type 2 macrophages (M2). ${ }^{4}$ M1 macrophages, also known as classically activated macrophages, are activated by Th1 cytokines to promote the response of inflammatory and the activities of anti-tumor. On the contrary, M2 macrophages (labeled by CD163, CD204, CD206, and so on), also known as alternatively activated macrophages, are activated by $\mathrm{Th} 2$ cytokines, which play a critical role in the pro-tumor activities such as anti-inflammatory, tissue remodeling, tumor proliferation, invasion, and metastasis.

Tumor-associated macrophages are now recognized as prognostic indicators in many solid tumors. A few studies verified that the high expression of TAMs associated with poor prognosis in gastric cancer, ${ }^{5}$ ovarian cancer, ${ }^{6}$ and nonsmall-cell lung cancer. ${ }^{7}$ But an increased density of TAMs in the invasive front of colon cancer associated with favorable prognosis. ${ }^{8}$ However, the impact of tumor-associated macrophages on patient prognosis in pancreatic cancer still exists controversy among previous studies. ${ }^{9-11}$ What's more, the biomarkers of tumor-associated macrophages in each study are not the same and the numbers of samples from the patients in each study variated. In order to solve these problems, we performed a meta-analysis to evaluate the potential association between TAMs density and the prognosis of pancreatic cancer.

\section{Materials and methods}

We conducted this meta-analysis based on the PRISMA guidelines. Ethical approval and informed consent are not needed because of the reason that the studies included in this meta-analysis are previously published.

\section{Search strategy}

We retrieved related articles about the prognostic value of TAMs in patients with pancreatic cancer, which was published before November 2018, from PubMed databases, Embase and Web of Science. The following search terms were variously combined: "pancreatic cancer", "pancreatic carcinoma", "pancreatic duct adenocarcinoma", "pancreatic ductal adenocarcinoma", "macrophage", "tumor-associated-macrophages", "tumor-infiltrating-macrophages", "tumor infiltrating macrophages", and "TAMs". The full electronic strategy of databases is listed in Supplementary text 1 . To identify potentially eligible articles, we checked titles and abstracts, and then reviewed full texts. What's more, we also manually checked the reference entries of the relevant literature to avoid the omission.

\section{Inclusion criteria and exclusion criteria}

The articles were reviewed by two authors. And disagreements were reached consensus by discussion. The study was included in this meta-analysis if meeting the following inclusion criteria: (1) being published in the English language, (2) researching pancreatic cancer patients, (3) investigating the information of TAMs expression, and (4) focusing on the association between TAMs and survival rates of patients with pancreatic cancer. Articles were excluded if meeting any of the following exclusion criteria: (1) not analyzing the prognosis value of pancreatic cancer; (2) not providing sufficient data to extract HRs and $95 \%$ confidence intervals (95\% CIs); (3) reporting duplicate or overlapping data; (4) being specific literature such as animal studies, case reports, comments, reviews, and meeting abstracts; and (5) not full-text.

\section{Outcomes of interest}

The foremost outcomes of interest in our meta-analysis were overall survival (OS), disease-free survival (DFS), and relapse-free survival (RFS). Other outcomes of interest were lymph node (LN) metastasis, tumor stage, histological grade, sex, and tumor location.

\section{Evaluation of the publication's quality}

We evaluated the quality of the original publications of three components: patient selection, study comparability and outcome assessment in the Newcastle-Ottawa scale (NOS) system. ${ }^{12}$ If a study obtained a score over 6 , it can be regarded as a study of high equality. Two authors independently performed evaluation of the publications quality and the disagreements were reached consensus by robust discussion.

\section{Data extraction}

The datum was extracted by two authors independently from included studies. The following data were extracted from each study: author, publication year, country, median age, the proportion of men and women, tissue distribution, cut-off value, disease stage, duration of follow-up, examination methods; biomarkers, the proportion of positive outcomes and negative outcomes, estimate HRs, low limit of $95 \%$ confidence interval(LCI) and upper limit of 95\% confidence interval (UCI) concerning the prognostic significance of TAMs, survival analysis, dilution and NOS score. HRs and their 95\% CIs were given directly in most of the original studies. Because of the fact that a few included studies presented survival curves rather than the 
HRs, hence we made use of the Engauge Digitizer 4.1 software to calculate HRs. ${ }^{13}$

\section{$\mathrm{HR}$ and $95 \% \mathrm{Cl}$ statistical analysis}

Using HRs with 95\% CIs, we investigate the association between the TAMs density and prognosis of patients with pancreatic cancer according to Tierney's method. ${ }^{14}$ Using Odds ratios (ORs) with CIs, we evaluated the correlation between the TAMs density and clinicopathological characteristics. The pooled results were presented with forest plots. If a pooled HR or OR is $>1$, it suggested there was a worse prognosis in the high TAMs density group. And if the $95 \%$ CI did not include 1, it was generally thought to indicate a statistically significant result. If a pooled HR or $\mathrm{OR}$ is $<1$, it suggested there was a better prognosis in the high TAMs density group.

Heterogeneity among included studies was assessed by the Cochran's Q statistic and $\mathrm{I}^{2}$ tests. According to Higgins and Thompson (2003), the heterogeneity was defined as either $\mathrm{I}^{2}>50 \%$ or $P$-value $<0.10$, in which case a random effect model would be used. If heterogeneity was not significant, we would choose a fixed effect model. We also conducted a subgroup analysis to seek the source of heterogeneity existing across studies. ${ }^{15}$ Meanwhile, by omitting a study at a time, a sensitivity analysis was performed to assess the stability. In addition, we performed Begg's test and Egger's test ${ }^{16}$ to confirm whether publication bias existed among the included publications. All the statistical analysis was based on STATA version 11 . These processes were performed by two authors independently.

\section{Results}

\section{Search results and characteristics of the study}

According to the inclusion criteria and exclusion criteria detailed in methods, 13 studies with macrophages detected by immunohistochemistry (IHC) or polymerase chain reaction (PCR) in tumor samples were included for meta-analysis. The flow chart of the search strategy is shown in Figure 1.

All the included studies published in English. The total sample size for all records was 1699 , and the size ranged from 28 to $284 .^{9-11,17-26}$ Among them, 6 cases enrolled less than 100 patients $^{10,17-20,26}$ and 7 studies $^{9,11,21-25}$ included more than 100 patients. The NOS scores of these studies ranged from 6 to 8 . Thus, the quality of all included studies was good. Among these cases, the tissue distributions variated. First, seven cases detected TAMs in the tumor cell and stroma, and four cases focused on TAMs locating in tumor cell. Excepted that, two cases accessed TAMs locating in tumor

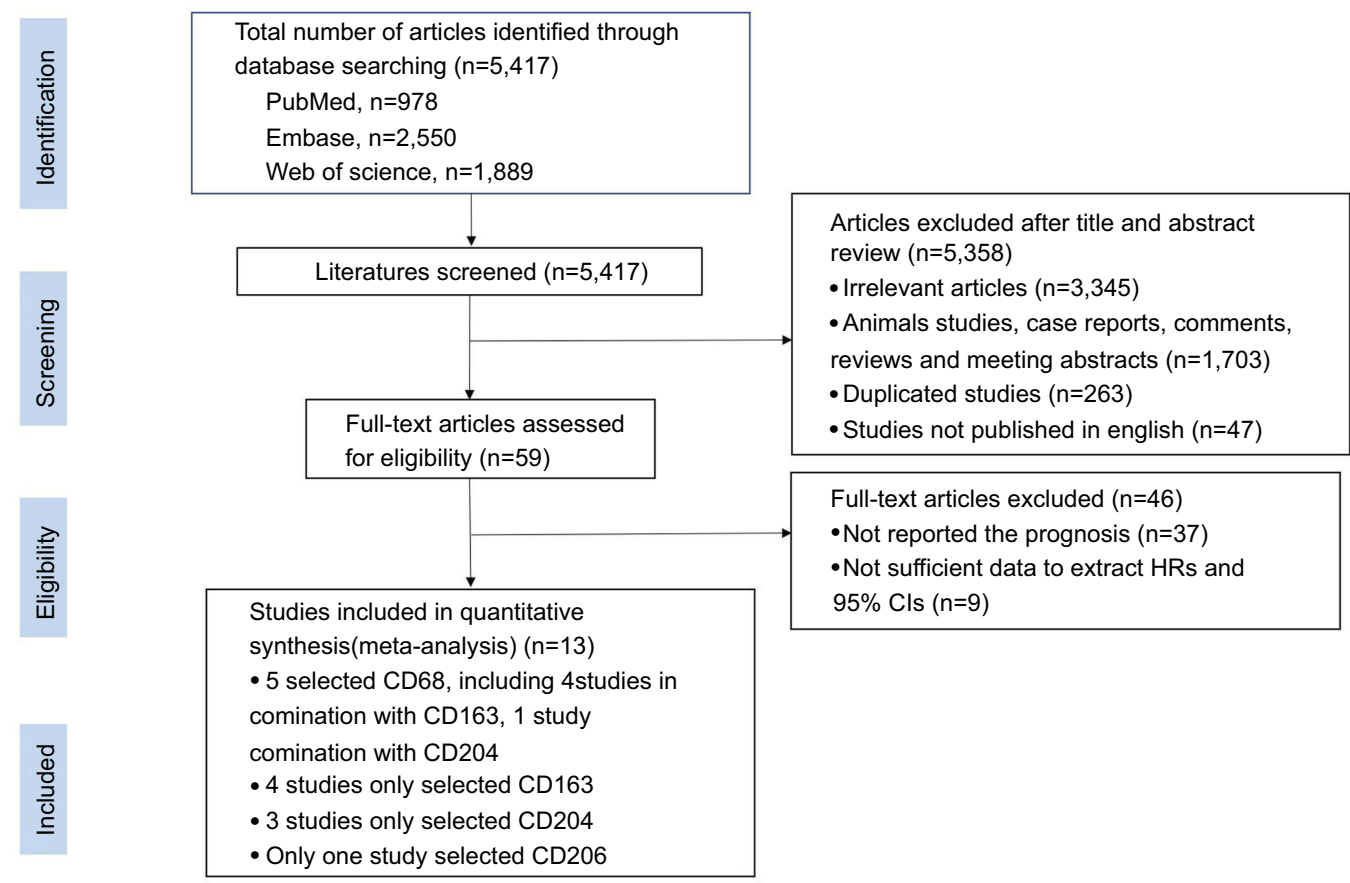

Figure I Flow diagram of study selection. 
cell. These studies enrolled patients from East Asia, Europe, and North America, including 5 from China, 5 from Japan, 2 from the USA, and 1 from Germany. When talking about prognostic endpoints in the included studies, OS, DFS, and RFS were used jointly or separately. Details of the characteristics of studies are shown in Table 1.

\section{Detection of tumor-associated macrophages}

Examination methods were mainly IHC, one of which combined with PCR. Multiple markers were used to estimate tumor-associated macrophages. CD68 was used for identification of TAMs in 5 of the 13 included studies, including 4 studies in combination with CD163, ${ }^{9,11,22,26} 1$ study in combination with CD204. ${ }^{10}$ Four studies only selected CD163 as a biomarker ${ }^{18-21}$ and 3 studies only selected CD204 $4^{17,22,24}$ as the biomarker. Only one study only selected CD206 as a biomarker. ${ }^{23}$ We also can see the details of multiple markers in Table 1. Stained sections were scanned at a magnification of X40 and authors marked the margin of the tumor on each slide. The center of lesion was defined as the intersection of the major and minor axes and four fields including the center at a magnification of X100. Peripheral site was defined as fields that included cancer cells and adjacent noncancerous cells at a magnification of X100. ${ }^{22}$

\section{The association of TAMs with clinicopathological characteristics}

The effect of elevated TAMs infiltration with the clinicopathological parameters in pancreatic cancer patients was further evaluated in our meta-analysis. Four of the 13 included studies have presented relevant data of the association between clinicopathological parameters and CD68+ TAMs. ${ }^{10,17,25,26}$ Three of the 13 included studies have presented relevant data of the association between clinicopathological parameters and CD204+ TAMs. Two of the 13 included studies have presented relevant data of the association between clinicopathological parameters and CD163+ TAMs. Moreover, there were three studies investigating the connection between M2 TAMs and clinicopathological parameters. It is a pity that there was not enough data for CD206+ TAMs. Pooled analysis showed no significant correlation between elevated CD68+ TAMs infiltration and LN metastasis (yes vs no) $(\mathrm{OR}=1.45,95 \% \mathrm{CI}$ $=0.89-2.39$, fixed effects model, $P=0.136)$, tumor stage $(\mathrm{T} 1 /$ $\mathrm{T} 2$ vs T3/T4) $(\mathrm{OR}=0.60,95 \% \mathrm{CI}=0.36-1.02$, fixed effects model, $P=0.061$ ), histological grade (III vs I, II)
$(\mathrm{OR}=0.75,95 \%$ CI $=0.39-1.45$, fixed effects model, $P=0.394)$, sex (male vs female) $\quad(\mathrm{OR}=0.69,95 \%$ $\mathrm{CI}=0.43-1.10$, fixed effects model, $P=0.120$ ), or tumor location (head/neck vs other) $(\mathrm{OR}=1.12,95 \% \mathrm{CI}=0.47-2.68$, fixed effects model, $P=0.730)$. Pooled analysis showed no significant correlation between elevated CD163+ TAMs infiltration and LN metastasis (yes vs no) $(\mathrm{OR}=2.69,95 \% \mathrm{CI}$ $=0.52-14.03$, random effects model, $P=0.241)$, tumor stage $(\mathrm{T} 1 / \mathrm{T} 2 \mathrm{vs} \mathrm{T} 3 / \mathrm{T} 4)(\mathrm{OR}=0.25,95 \% \mathrm{CI}=0.03-2.27$, fixed effects model, $P=0.219)$, histological grade (III vs I, II) $(\mathrm{OR}=3.00$, $95 \% \mathrm{CI}=0.51-17.50$, fixed effects model, $P=0.222$ ), sex (male vs female) ( $\mathrm{OR}=1.09,95 \% \mathrm{CI}=0.56-2.12$, fixed effects model, $P=0.802$ ), or tumor location (head/neck vs other) (OR $=0.51,95 \%$ CI $=0.21-1.27$, fixed effects model, $P=0.151$ ). Pooled analysis showed no significant correlation between elevated CD204+ TAMs infiltration and LN metastasis (yes vs no) $(\mathrm{OR}=1.915,95 \% \mathrm{CI}=0.26-13.94$, random effects model, $P=0.525)$, tumor stage (T1/T2 vs T3/T4) $(\mathrm{OR}=0.72$, $95 \% \mathrm{CI}=0.27-1.95$, fixed effects model, $P=0.518)$, histological grade (III vs I, II) $(\mathrm{OR}=1.33,95 \% \mathrm{CI}=0.31-5.67$, random effects model, $P=0.801)$, sex (male vs female) $(\mathrm{OR}=1.10,95 \%$ $\mathrm{CI}=0.58-2.11$, fixed effects model, $P=0.771$ ), or tumor location (head/neck vs other) $(\mathrm{OR}=1.17,95 \% \mathrm{CI}=0.48-2.87$, fixed effects model, $P=0.724$ ) as well. The same result also happened when we investigated M2-TAMs. The details about the association of TAMs with clinicopathological characteristics are shown in Tables 2-5.

\section{Association of M2-TAMs with the survival of pancreatic cancer}

As we can see in Figure 2A, the pooled results of eleven studies showed that patients with high M2-TAMs infiltration had significantly decreased OS compared to those with low/negative M2-TAMs infiltration ( $\mathrm{HR}=1.95,95 \% \mathrm{CI}=1.63-2.33$, random effects model, $\mathrm{I}^{2}=59.2 \%, P=0.000$ ). Furthermore, a subgroup analysis according to the M2-TAMs in different sample distribution also showed that the high M2-TAMs infiltration indicated a significant correlation with worse OS in pancreatic cancer patients whose biomarkers were detected in tumor cell and stroma (HR $=1.89,95 \%$ CI $=1.39-2.56$, $P=0.000$ ) (Figure $\mathrm{S} 1 \mathrm{~A}$, distribution $=$ tumor cell and stroma), tumor stroma $(\mathrm{HR}=2.19,95 \% \mathrm{CI}=1.65-2.90, P=0.000)$ (Figure S1A, distribution $=$ stroma), and tumor cell $(\mathrm{HR}$ $=1.80,95 \% \mathrm{CI}=1.29-2.50, P=0.001$ ) (Figure $\mathrm{S} 1 \mathrm{~A}$, distribution $=$ tumor cell). We also conducted a subgroup analysis among the pancreatic cancer patients, according to the country. The pooled HRs demonstrated M2-TAMs correlated with 


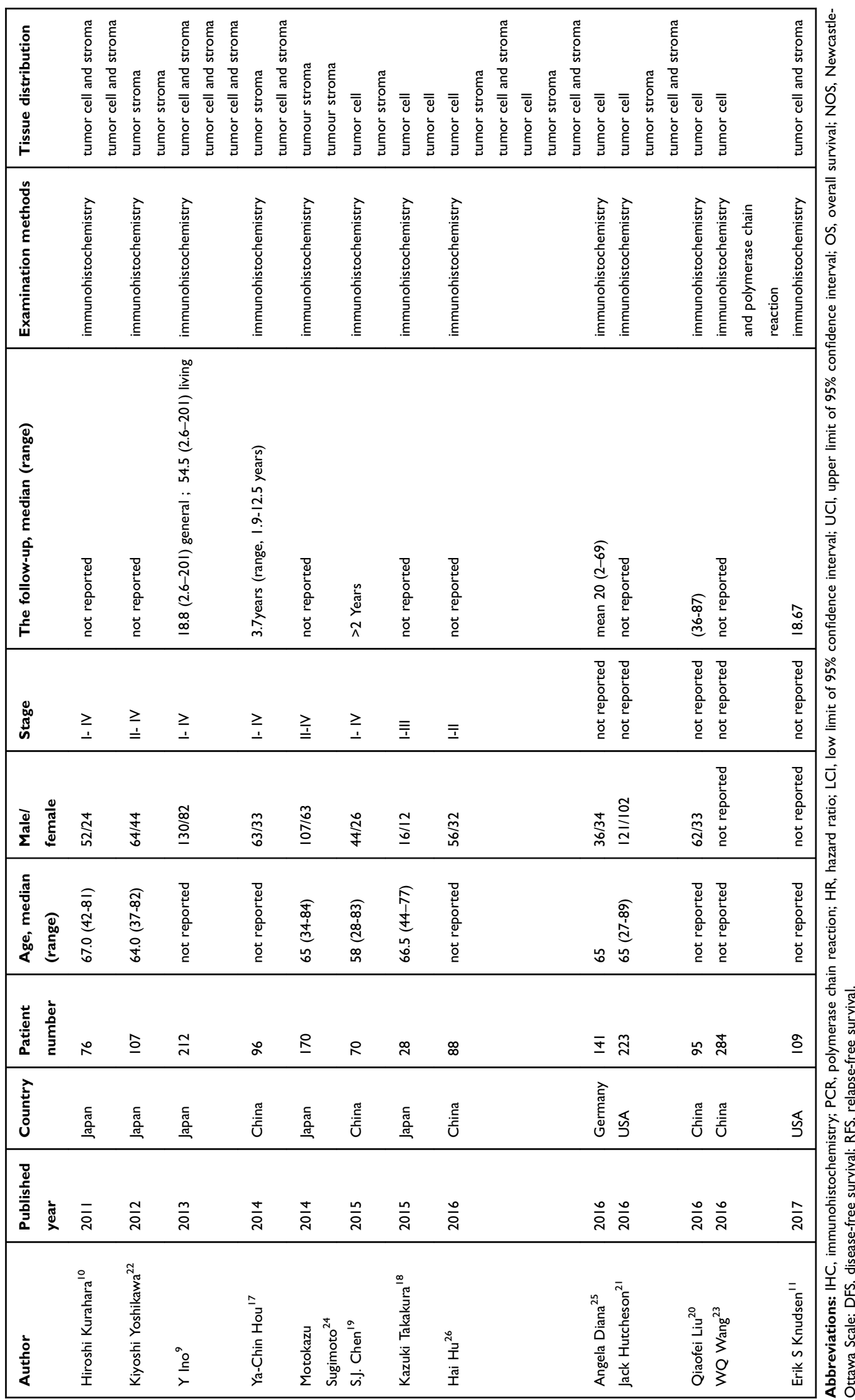




\begin{tabular}{|c|c|c|c|c|c|c|c|c|c|c|c|c|c|c|c|}
\hline 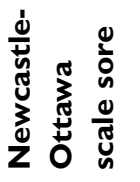 & $\wedge$ & & $n$ & & $n$ & & & r & & 0 & & $\infty$ & & $\infty$ & \\
\hline 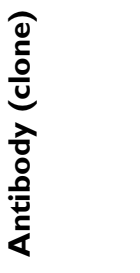 & 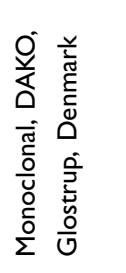 & 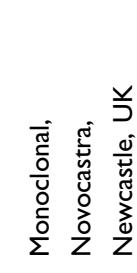 & 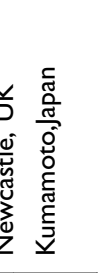 & 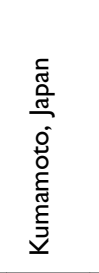 & 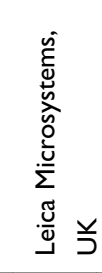 & 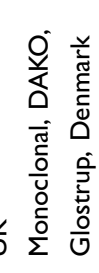 & 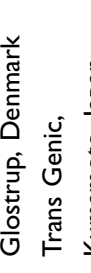 & 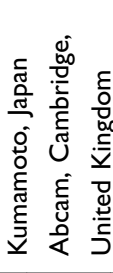 & 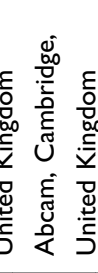 & 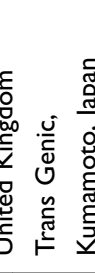 & 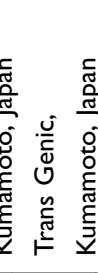 & 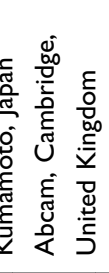 & 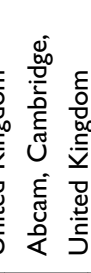 & 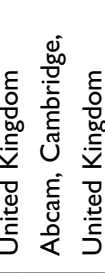 & 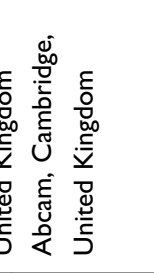 \\
\hline$\frac{0}{\stackrel{0}{2}}$ & $\stackrel{8}{\underline{-}}$ & $\begin{array}{l}\stackrel{\circ}{n} \\
\stackrel{-}{-}\end{array}$ & 吕 & 祦 & 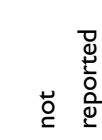 & 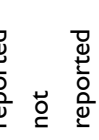 & 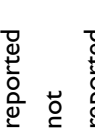 & 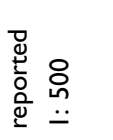 & $\begin{array}{l}\stackrel{8}{\circ} \\
\stackrel{\leftrightarrow}{-}\end{array}$ & $\begin{array}{l}\stackrel{8}{+} \\
\stackrel{-}{-}\end{array}$ & $\begin{array}{l}\stackrel{8}{+} \\
\stackrel{-}{-}\end{array}$ & $\begin{array}{l}\stackrel{8}{\circ} \\
\stackrel{\leftrightarrow}{-}\end{array}$ & $\begin{array}{l}\stackrel{8}{\circ} \\
\stackrel{-}{-}\end{array}$ & $\begin{array}{l}\stackrel{8}{\circ} \\
\stackrel{\circ}{-}\end{array}$ & $\begin{array}{l}\stackrel{8}{\circ} \\
\stackrel{\leftrightarrow}{-}\end{array}$ \\
\hline 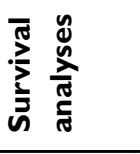 & 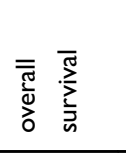 & 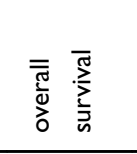 & 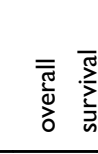 & $\begin{array}{l}\bar{y} \\
\overline{0} \\
\overline{0}\end{array}$ & 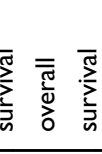 & 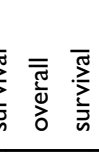 & 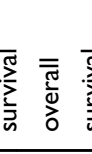 & 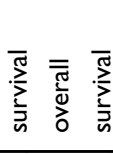 & 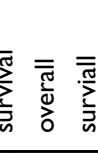 & 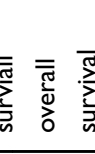 & 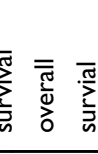 & 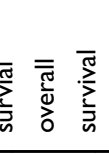 & 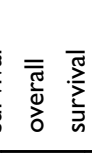 & 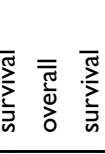 & 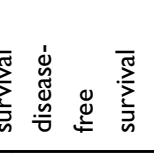 \\
\hline 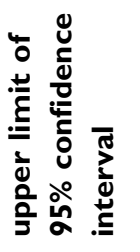 & 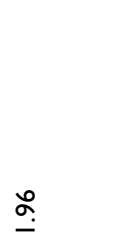 & $\stackrel{\llcorner}{\text { nִ }}$ & $\underset{\substack{\text { fo } \\
\dot{r}}}{ }$ & 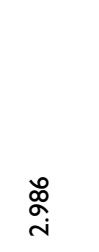 & 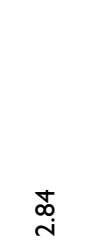 & $\underset{\sim}{\mathbb{n}}$ & $\stackrel{\infty}{\stackrel{\infty}{-}}$ & $\begin{array}{l}\stackrel{2}{a} \\
\dot{+}\end{array}$ & $\underset{\sim}{\tilde{m}}$ & 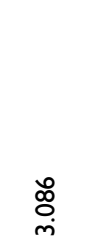 & 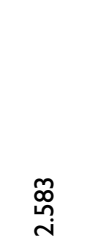 & $\stackrel{\sim}{m}$ & $\bar{ঙ}$ & $\frac{\stackrel{m}{m}}{\dot{\dot{I}}}$ & $\underset{\substack{n \\
\infty}}{a}$ \\
\hline 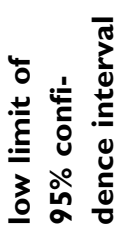 & nุ. & $\stackrel{\tilde{m}}{\underline{.}}$ & $\stackrel{ \pm}{\stackrel{ \pm}{I}}$ & 志 & $\stackrel{0}{1}$ & 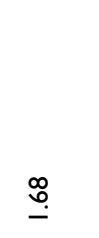 & 무. & $\begin{array}{l}\infty \\
\stackrel{\infty}{0} \\
\stackrel{-}{-}\end{array}$ & ঃั & הี & సે & $\equiv$ & 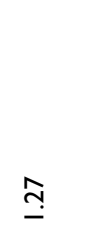 & $\stackrel{\infty}{\stackrel{\infty}{-}}$ & 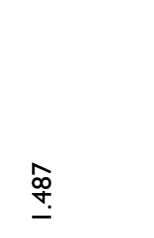 \\
\hline 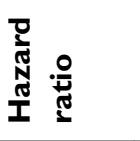 & - & $\frac{n}{i}$ & $\stackrel{\bar{\infty}}{\stackrel{i}{i}}$ & 䓌 & $\stackrel{m}{\text { in }}$ & $\stackrel{m}{\sim}$ & $\stackrel{\infty}{\rightarrow}$ & 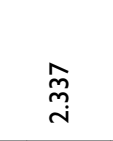 & $\stackrel{\infty}{-}$ & $\frac{\hat{0}}{i}$ & $\stackrel{\overline{0}}{\underline{\infty}}$ & $\stackrel{\infty}{\stackrel{\infty}{-}}$ & $\bar{M}$ & $\begin{array}{l}\stackrel{+}{\infty} \\
\stackrel{+}{+}\end{array}$ & 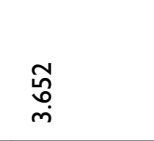 \\
\hline 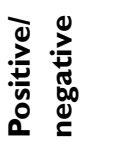 & $\stackrel{\stackrel{2}{S}}{\text { ก }}$ & $\underset{\mathcal{F}}{\mathcal{f}}$ & $\stackrel{+}{\stackrel{H}{N}}$ & 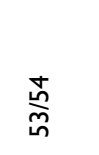 & $\frac{\circ}{\frac{0}{o}}$ & 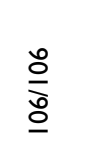 & $\frac{\circ}{\frac{0}{o}}$ & 会 & $\stackrel{n}{\stackrel{n}{\rho}}$ & 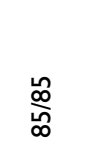 & 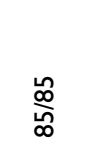 & $\underset{\substack{\stackrel{m}{f} \\
\text { m }}}{ }$ & $\underset{m}{\stackrel{m}{i}}$ & $\frac{\vec{\sigma}}{\vec{\theta}}$ & $\frac{\vec{J}}{\dot{\sigma}}$ \\
\hline$\frac{\grave{d}}{\frac{\grave{v}}{2}}$ & 号 & $\frac{\tilde{\circ}}{0}$ & 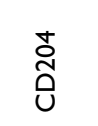 & 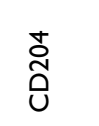 & $\frac{\tilde{0}}{0}$ & 号 & 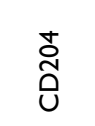 & 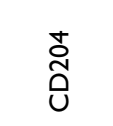 & Oิ̊ & 苂 & 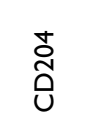 & $\frac{m}{0}$ & $\frac{\tilde{6}}{0}$ & อิ & $\frac{n}{0}$ \\
\hline 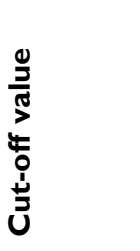 & 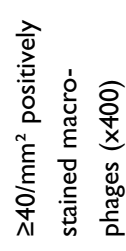 & 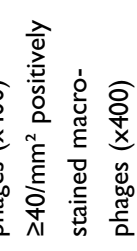 & 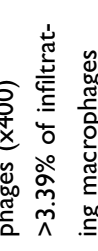 & 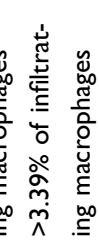 & 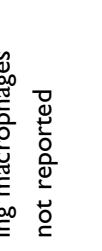 & 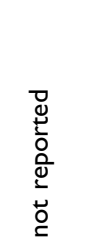 & 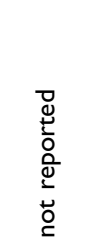 & $\begin{array}{l}\stackrel{\widetilde{\varpi}}{\Sigma} \\
\sum\end{array}$ & $\stackrel{\text { 厄్ }}{\Sigma}$ & 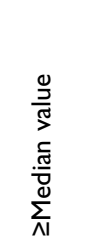 & 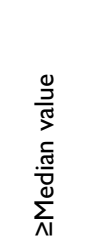 & 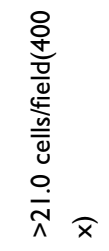 & 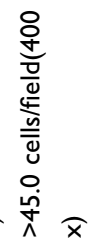 & 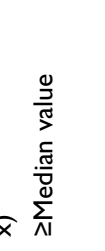 & 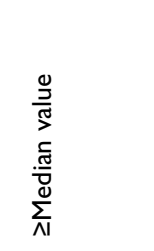 \\
\hline 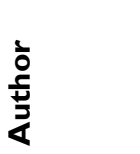 & 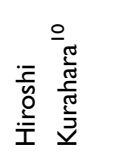 & & 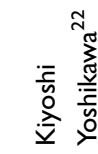 & & $\frac{\circ}{\stackrel{\circ}{\underline{\rho}}}$ & & & 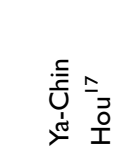 & & 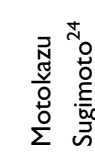 & & $\begin{array}{l}\frac{\sigma}{\bar{c}} \\
\bar{\delta} \\
\dot{\delta} \\
\dot{v}\end{array}$ & & 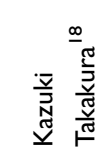 & \\
\hline
\end{tabular}




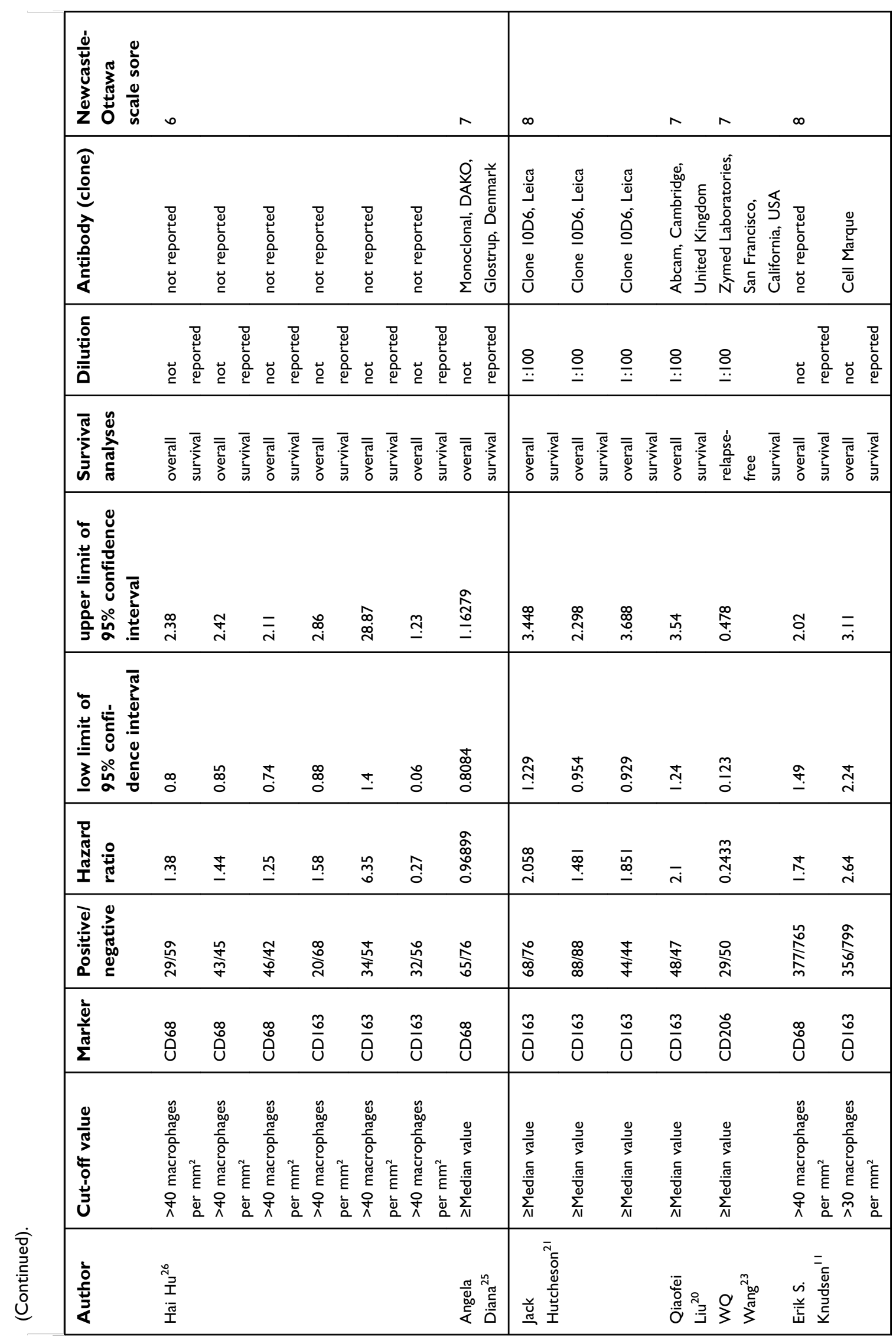


Table 2 Meta-analysis of high CD68+ TAMs infiltration and clinicopathological features of pancreatic cancer patients

\begin{tabular}{|l|l|l|l|l|l|l|}
\hline Patient characteristics & \multicolumn{3}{|l|}{$\begin{array}{l}\text { Odds ratio (95\% confidence } \\
\text { interval) }\end{array}$} & Heterogeneity & \\
\hline (CD68 High vs Low) & $\begin{array}{l}\text { References } \\
\text { of studies }\end{array}$ & $\begin{array}{l}\text { Number of } \\
\text { patients }\end{array}$ & Fixed & Random & $\mathbf{I}^{\mathbf{2}}$ & P-value \\
\hline $\begin{array}{l}\text { Lymph node metastasis } \\
\text { (yes vs no) }\end{array}$ & $10,26,25$ & 305 & $1.45(0.89-2.39)$ & $1.49(0.81-2.73)$ & $32.00 \%$ & 0.23 \\
$\begin{array}{l}\text { Tumor stage } \\
\text { (TI/T2 vs T3/T4) }\end{array}$ & $10,17,26,25$ & 401 & $0.60(0.36-1.02)$ & $0.61(0.36-1.03)$ & 0 & 0.67 \\
$\begin{array}{l}\text { Histological grade (III vs I, II) } \\
\text { Sex (male vs female) }\end{array}$ & 10,26 & 216 & $0.75(0.39-1.45)$ & $0.75(0.39-1.45)$ & 0 \\
Tumor location (head/neck vs \\
other)
\end{tabular}

Table 3 Meta-analysis of high M2-TAMs infiltration and clinicopathological features of pancreatic cancer patients

\begin{tabular}{|c|c|c|c|c|c|c|}
\hline \multirow{2}{*}{$\begin{array}{l}\text { Patient characteristics } \\
\text { (Type } 2 \text { macrophages } \\
\text { High vs Low) }\end{array}$} & \multirow[b]{2}{*}{$\begin{array}{l}\text { References } \\
\text { of studies }\end{array}$} & \multirow[b]{2}{*}{$\begin{array}{l}\text { Number of } \\
\text { patients }\end{array}$} & \multicolumn{2}{|c|}{$\begin{array}{l}\text { Odds ratio ( } 95 \% \text { confidence } \\
\text { interval) }\end{array}$} & \multirow{2}{*}{$\begin{array}{l}\text { Heterogeneity } \\
\mathbf{I}^{2}\end{array}$} & \multirow[b]{2}{*}{$P$-value } \\
\hline & & & Fixed & Random & & \\
\hline $\begin{array}{l}\text { Lymph node metastasis (yes } \\
\text { vs no) }\end{array}$ & $10,17,26$ & 260 & $1.90(1.21-2.98)$ & $2.22(0.76-6.46)$ & $79.20 \%$ & 0.002 \\
\hline Tumor stage (TI/T2 vs $\mathrm{T} 3 / \mathrm{T} 4)$ & 10,26 & 164 & $0.58(0.24-1.39)$ & $0.63(0.24-1.61)$ & 0 & 0.392 \\
\hline Histological grade (III vs I, II) & 10,17 & 172 & $1.37(0.63-2.98)$ & $1.57(0.54-4.55)$ & $36.00 \%$ & 0.21 \\
\hline Sex (male vs female) & $10,17,26$ & 260 & $1.09(0.69-1.74)$ & $1.09(0.69-1.74)$ & 0 & 0.888 \\
\hline $\begin{array}{l}\text { Tumor location (head/neck vs } \\
\text { other) }\end{array}$ & 10,17 & 184 & $0.79(0.42-1.48)$ & $0.78(0.35-1.75)$ & $38.10 \%$ & 0.204 \\
\hline
\end{tabular}

Table 4 Meta-analysis of high CDI63+ TAMs infiltration and clinicopathological features of pancreatic cancer patients

\begin{tabular}{|c|c|c|c|c|c|c|}
\hline \multirow{2}{*}{$\begin{array}{l}\text { Patient characteristics } \\
\text { (CDI63 High vs Low) }\end{array}$} & \multirow[b]{2}{*}{$\begin{array}{l}\text { References } \\
\text { of studies }\end{array}$} & \multirow[b]{2}{*}{$\begin{array}{l}\text { Number of } \\
\text { patients }\end{array}$} & \multicolumn{2}{|c|}{$\begin{array}{l}\text { Odds ratio ( } 95 \% \text { confidence } \\
\text { interval) }\end{array}$} & \multirow{2}{*}{$\begin{array}{l}\text { Heterogeneity } \\
I^{2}\end{array}$} & \multirow[b]{2}{*}{$P$-value } \\
\hline & & & Fixed & Random & & \\
\hline $\begin{array}{l}\text { Lymph node metastasis (yes } \\
\text { vs no) }\end{array}$ & 10,26 & 164 & $2.28(1.14-4.56)$ & $2.69(0.52-14.03)$ & $81.40 \%$ & 0.021 \\
\hline Tumor stage (TI/T2 vs T3/T4) & 10 & 76 & $0.25(0.03-2.27)$ & $0.25(0.03-2.27)$ & & \\
\hline Histological grade (III vs I, II) & 10 & 76 & $3.00(0.5 I-17.50)$ & $3.00(0.5 I-17.50)$ & & \\
\hline Sex (male vs female) & 10,26 & 164 & $1.09(0.56-2.12)$ & $1.09(0.56-2.12)$ & 0 & 0.602 \\
\hline $\begin{array}{l}\text { Tumor location (head/neck vs } \\
\text { other) }\end{array}$ & 10 & 88 & $0.5 \mathrm{I}(0.2 \mathrm{I}-\mathrm{I} .27)$ & $0.5 I(0.2 I-\mid .27)$ & . & \\
\hline
\end{tabular}

worse OS in pancreatic cancer patients in the non-Asian country $(\mathrm{HR}=2.08,95 \% \mathrm{CI}=1.52-2.83, P=0.000)$ (Figure $\mathrm{S} 1 \mathrm{~B})$. And there was a significant connection between CD163+ TAMs and worse OS in pancreatic cancer patients in the Asian country $(\mathrm{HR}=1.91,95 \% \mathrm{CI}=1.55-2.36, P=0.000)$ (Figure S1B). Based on the number of patients in studies, a subgroup analysis, in which studies were divided into two groups, was conducted. The pooled data indicated that M2TAMs correlated with worse OS in pancreatic cancer patients in the studies whose patient number were below 100 (HR $=2.05,95 \% \mathrm{CI}=1.58-2.67, P=0.000$ ) (Figure S1C). There was a significant connection between M2-TAMs and worse 
Table 5 Meta-analysis of high CD204+ TAMs infiltration and clinicopathological features of pancreatic cancer patients

\begin{tabular}{|c|c|c|c|c|c|c|}
\hline \multirow{2}{*}{$\begin{array}{l}\text { Patient characteristics } \\
\text { (CD204 High vs Low) }\end{array}$} & \multirow[b]{2}{*}{$\begin{array}{l}\text { References } \\
\text { of studies }\end{array}$} & \multirow[b]{2}{*}{$\begin{array}{l}\text { Number of } \\
\text { patients }\end{array}$} & \multicolumn{2}{|c|}{$\begin{array}{l}\text { Odds ratio ( } 95 \% \text { confidence } \\
\text { interval) }\end{array}$} & \multirow{2}{*}{$\begin{array}{l}\text { Heterogeneity } \\
\mathbf{I}^{2}\end{array}$} & \multirow[b]{2}{*}{$P$-value } \\
\hline & & & Fixed & Random & & \\
\hline $\begin{array}{l}\text { Lymph node metastasis (yes vs } \\
\text { no) }\end{array}$ & 10,17 & 172 & $\mathrm{I} .45(0.75-2.8 \mathrm{I})$ & $1.91(0.26-13.94)$ & $87.90 \%$ & 0.004 \\
\hline Tumor stage (TI/T2 vs T3/T4) & 10,26 & 164 & $0.72(0.27-1.95)$ & $0.76(0.25-2.26)$ & 0 & 0.756 \\
\hline Histological grade (III vs I, II) & 10,17 & 172 & $1.12(0.47-2.68)$ & $1.33(0.31-5.67)$ & $52.90 \%$ & 0.145 \\
\hline Sex (male vs female) & 10,17 & 172 & $1.10(0.58-2.11)$ & $1.10(0.58-2.11)$ & 0 & 0.545 \\
\hline $\begin{array}{l}\text { Tumor location (head/neck vs } \\
\text { other) }\end{array}$ & 17 & 96 & $1.17(0.48-2.87)$ & $1.17(0.48-2.87)$ & . & . \\
\hline
\end{tabular}
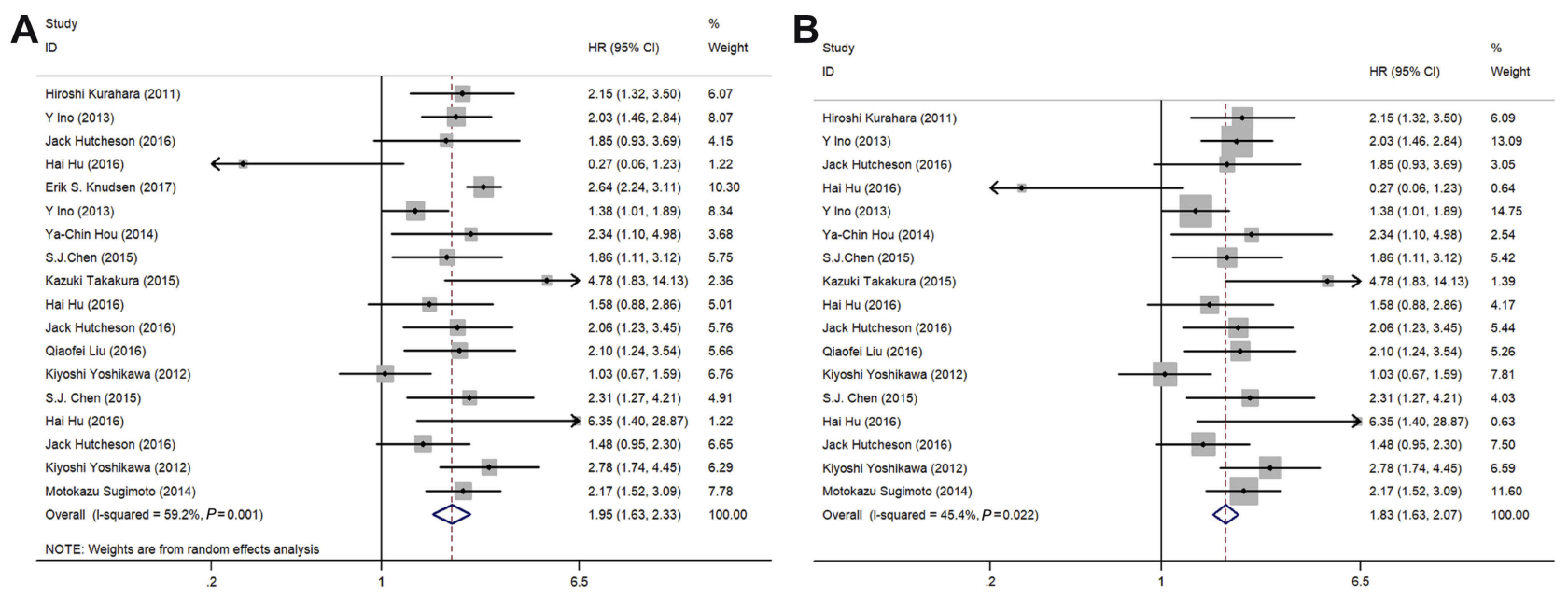

Figure 2 Forest plot of hazard ratio (HR) for the association between OS and M2-TAMs: all studies about M2-TAMs (A); all studies about M2-TAMs except the last included study (B).

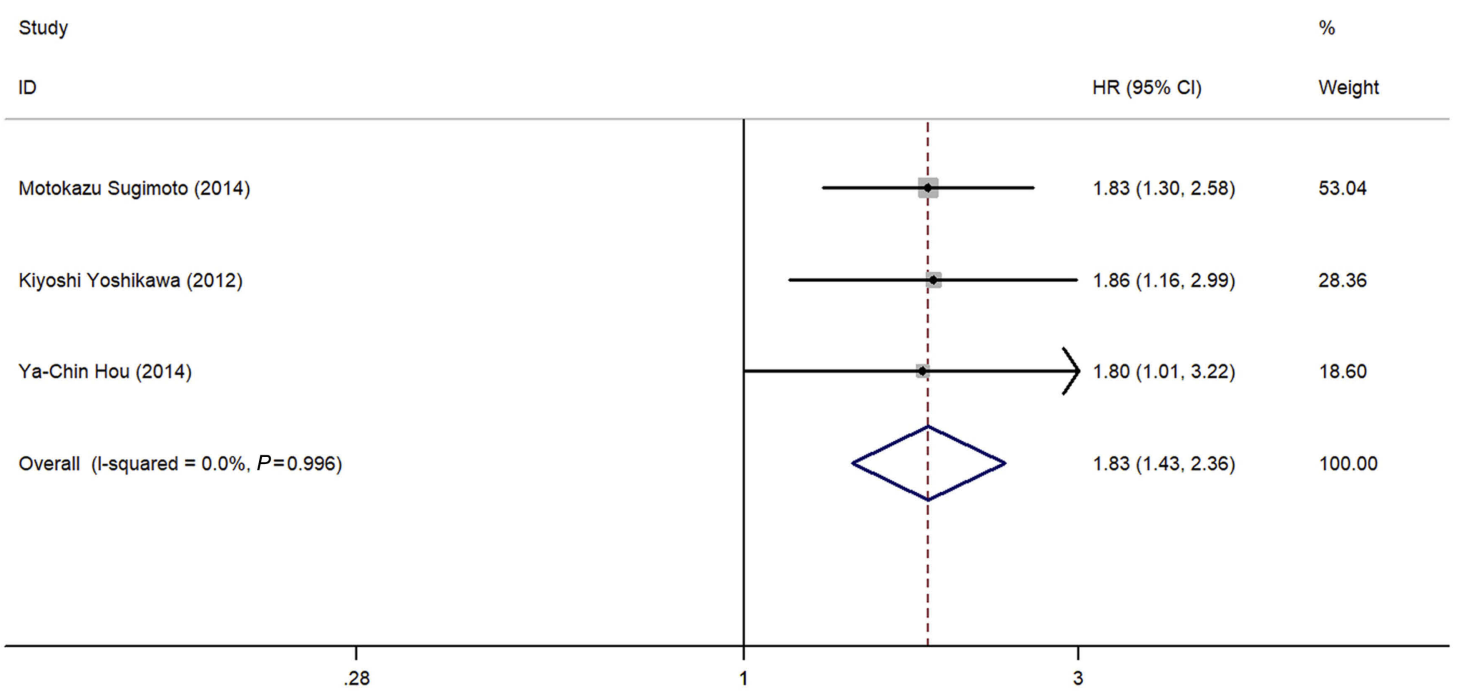

Figure 3 Forest plot of hazard ratio (HR) for the association between DFS and M2-TAMs. 


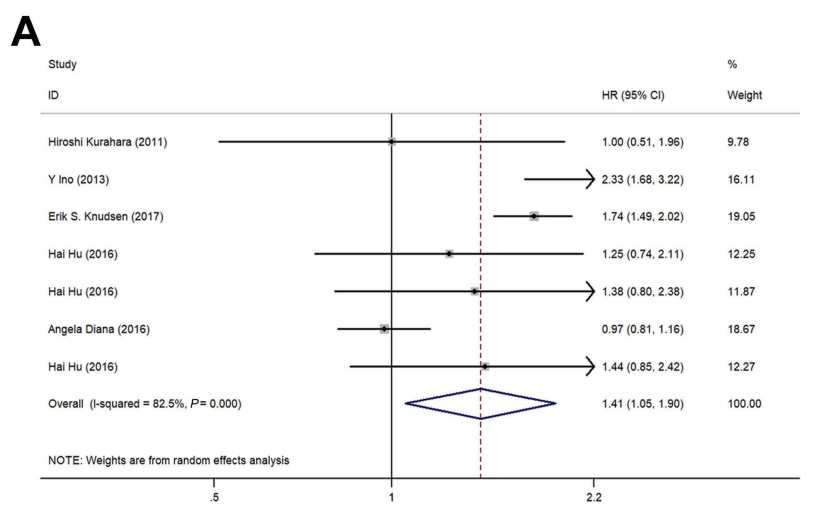

B

Figure 4 Forest plot of hazard ratio (HR) for the association between OS and CD68+ TAMs: all studies about CD68+ TAMs (A); all studies about CD68+ TAMs except the ninth included study and the last included study (B).

A

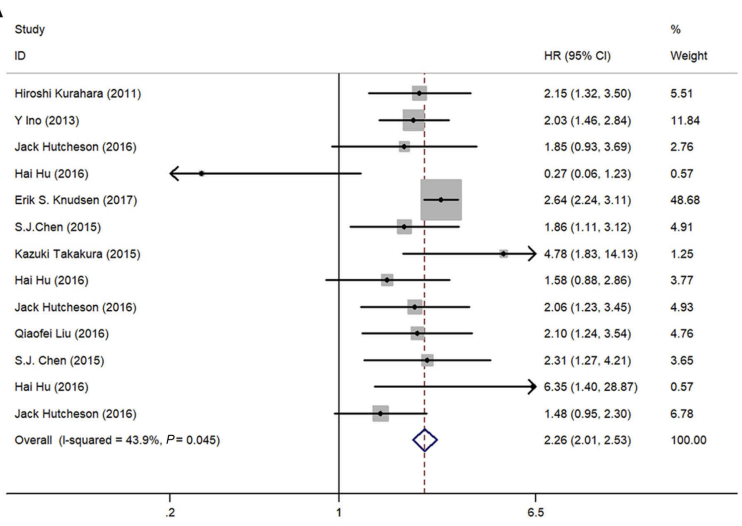

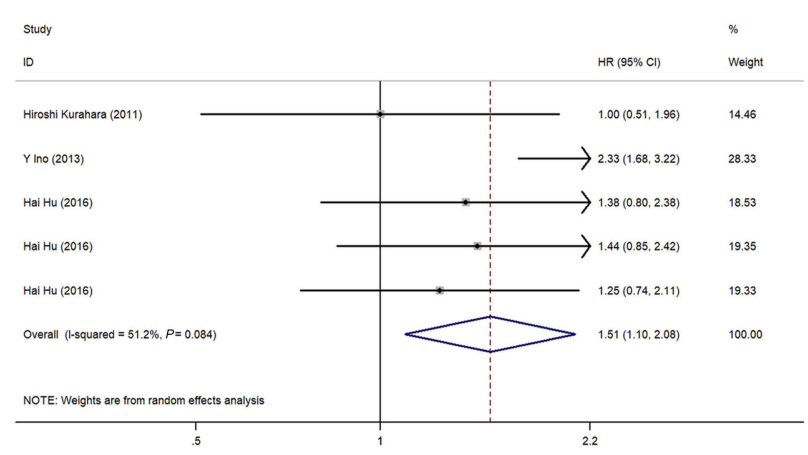

Figure 5 Forest plot of hazard ratio (HR) for the association between OS and CDI63+ TAMs: all studies about CDI63+ TAMs (A); all studies about CDI63+ TAMs except the last included study (B).

OS in pancreatic cancer patients in the studies whose patient number were above or equal to $100(\mathrm{HR}=1.88,95 \% \mathrm{CI}=1.47-$ 2.40, $P=0.000$ ) (Figure S1C). In order to seek the source of heterogeneity further, we conducted a subgroup analysis as well, according to the quality of studies. As shown in Figure S1D, the pooled HR showed that M2-TAMs correlated with worse OS in pancreatic cancer patients in the studies whose score were $7(\mathrm{HR}=1.83,95 \% \mathrm{CI}=1.41-2.36, P=0.000)$ and in pancreatic cancer patients in the studies whose score were 8 ( $\mathrm{HR}=2.18,95 \% \mathrm{CI}=1.75-2.73, P=0.000$ ). In contrast, there was a significant connection between M2-TAMs and worse OS in pancreatic cancer patients in the studies whose score were 6 $(\mathrm{HR}=1.69,95 \% \mathrm{CI}=0.83-3.44, P=0.151)$ (Figure S1D).

As for the prognostic value of M2-TAMs on DFS, the pooled results of three studies ${ }^{17,18,24}$ that elevated M2TAMs density was significantly associated with the DFS (HR $=1.83,95 \%$ CI $=1.43-2.36$, fixed effects model, $\mathrm{I}^{2}=0.0 \%, P=0.000$ ) (details in Figure 3).
B

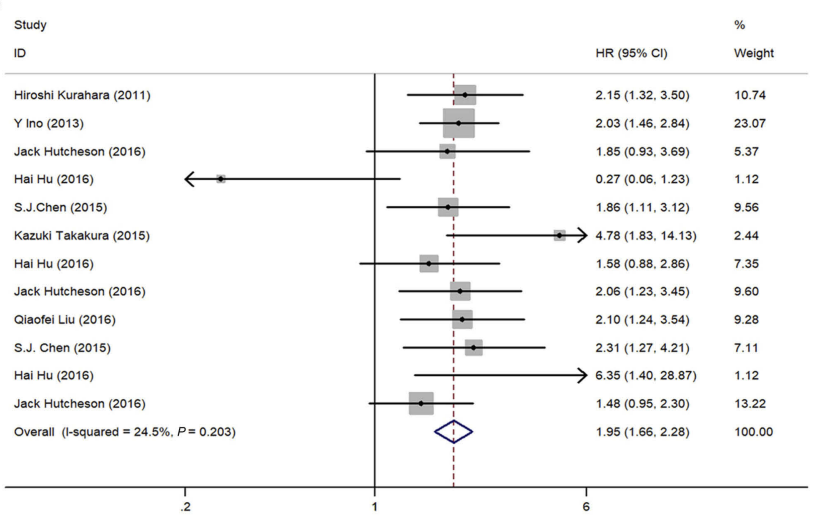

\section{The association between CD68+TAMs and prognostic outcomes}

To further investigate the prognostic role of CD68+ TAMs on OS, the pooled HRs demonstrated CD68+ TAMs correlated with worse $\mathrm{OS}$ in pancreatic cancer patients ( $\mathrm{HR}=1.41,95 \%$ $\mathrm{CI}=1.05-1.99$, random effects model, $\mathrm{I}^{2}=82.5 \%, P=0.021$ ) (Figure 4). We conducted a subgroup analysis according to the CD68+ TAMs in different sample distribution. Results demonstrated that there was no significant association between CD68+ TAMs and OS in pancreatic cancer patients whose $\mathrm{CD} 68+$ were detected in tumor cell $(\mathrm{HR}=1.05,95 \% \mathrm{CI}$ $=0.79-1.40, P=0.743$ ) (Figure S2A). The same result was also found when talking about the association between CD68+ TAMs and OS in pancreatic cancer patients whose CD68+ were identified in tumor stroma ( $\mathrm{HR}=1.44,95 \% \mathrm{CI}$ $=0.85-2.43, P=0.172$ ) (Figure S2A). In contrast, the pooled HRs demonstrated CD68+ TAMs correlated with worse OS in pancreatic cancer patients whose CD68+ were detected in 


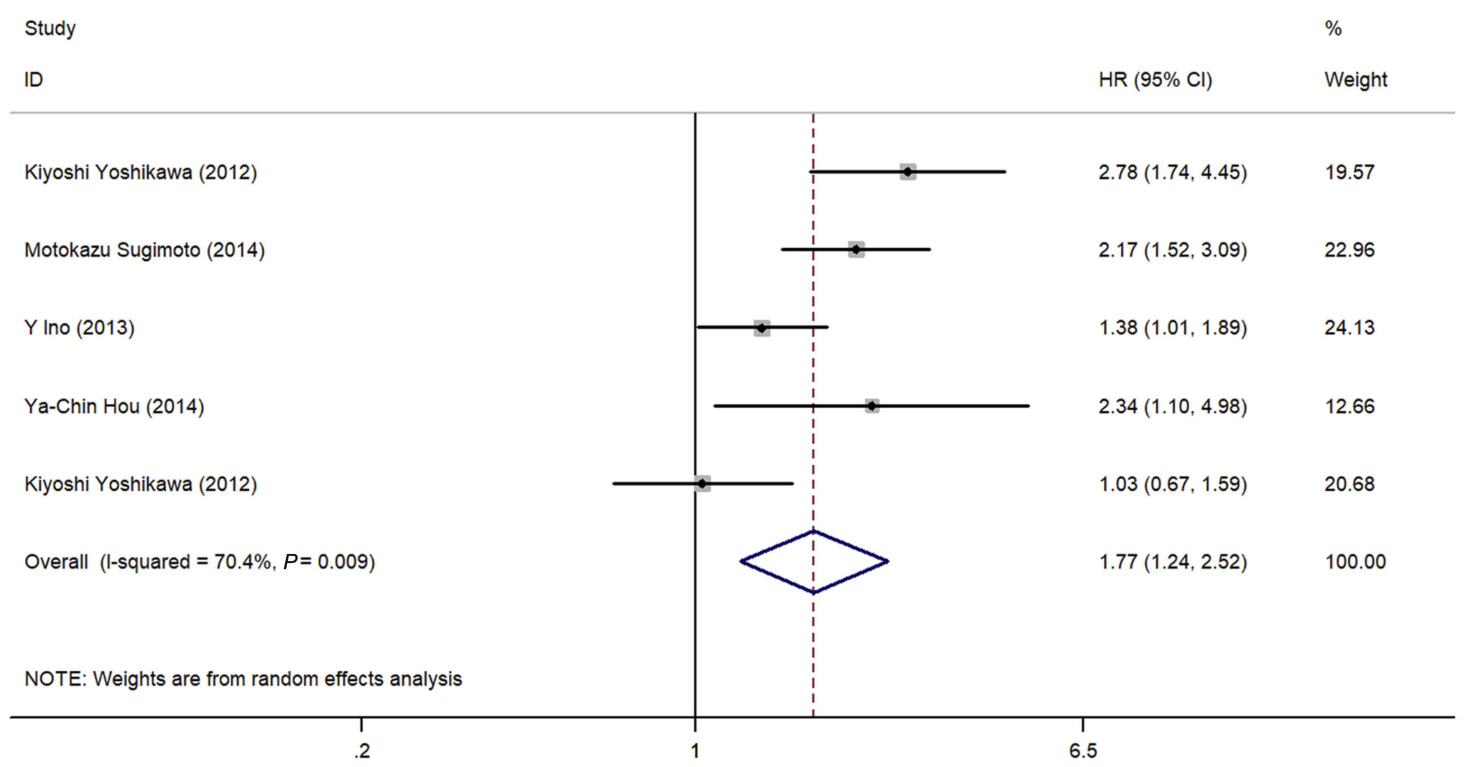

Figure 6 Forest plot of hazard ratio (HR) for the association between OS and CD204+ TAMs.

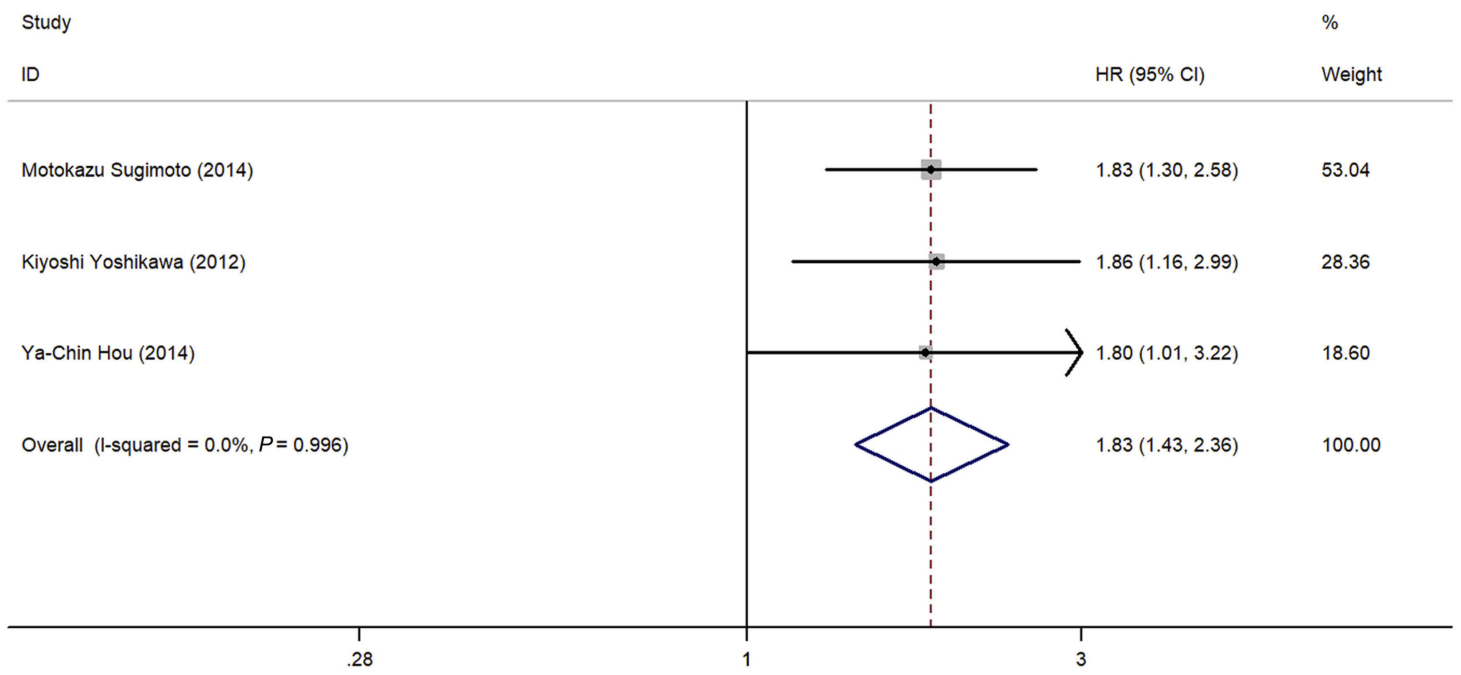

Figure 7 Forest plot of hazard ratio (HR) for the association between DFS and CD204+ TAMs.

tumor cell and stroma (HR $=1.66,95 \% \mathrm{CI}=1.25-2.20$, $P=0.000$ ) (Figure S2A)

\section{Prognostic significance of CDI63+TAMs in pancreatic cancer patients}

As for the prognostic value of CD163+ TAMs on OS, the pooled results of eight studies that elevated $\mathrm{CD} 163+$ density was significantly associated with the worse OS (HR $=2.26,95 \% \mathrm{CI}=2.01-2.53$, fixed effects model, $P=0.000$ ) and presented a significant degree of heterogeneity $\left(\mathrm{I}^{2}=43.9 \%, P=0.045\right)$ (details in Figure 5). In view of the existence of substantial heterogeneity, based on the CD163+ TAMs in different sample distribution, we conducted a subgroup analysis. The pooled HRs demonstrated CD163+ TAMs correlated with worse OS in pancreatic cancer patients, regardless of the $\mathrm{CD} 163+$ identified in tumor cell $(\mathrm{HR}=2.02,95 \% \mathrm{CI}=1.56-2.62, P=0.000)$ or in tumor stroma $(\mathrm{HR}=1.85,95 \% \mathrm{CI}=1.31-2.61, P=0.000)$ (Figure S2B). As for the OS in pancreatic cancer patients in reference to tumor cell and stroma, there was also significant connection with high CD163+ TAMs infiltration $(\mathrm{HR}=2.40, \quad 95 \% \quad \mathrm{CI}=2.09-2.76, \quad P=0.000)$ (Figure S2B). 


\section{Impact of CD204+TAMs on survival outcomes}

To further elucidate the role of CD204+ TAMs infiltration on survival outcomes, we investigated the correlation between CD204+ TAMs density and OS of pancreatic cancer patients. As shown in Figure 6, the pooled results of eight studies that elevated CD204+ density was significantly associated with the worse OS (HR $=1.77,95 \% \mathrm{CI}$ $=1.24-2.52$, random effects model, $P=0.002$ ) and presented a significant degree of heterogeneity $\left(\mathrm{I}^{2}=70.4 \%\right.$, $P=0.009$ ). A subgroup analysis was conducted by us according to the CD204+ TAMs in different sample distribution to seek for the source of substantial heterogeneity. The pooled HRs demonstrated CD204+ TAMs did not correlate with worse $\mathrm{OS}$ in pancreatic cancer patients whose CD204+ were detected tumor cell $(\mathrm{HR}=1.04$, $95 \%$ CI $=0.67-1.59, P=0.876$ ) (Figure $\mathrm{S} 2 \mathrm{C}$ ). As for the OS in pancreatic cancer patients whose CD204+ were identified in tumor cell and stroma, there was significant connection with high CD163+ TAMs infiltration (HR $=1.60$, 95\% CI $=1.01-2.54, P=0.047$ ) (Figure S2C). As for the OS in pancreatic cancer patients whose CD204+ were identified in tumor stroma, there was also significant connection with high CD204+ TAMs infiltration (HR $=2.37,95 \% \mathrm{CI}=1.79-3.15, P=0.000$ ) (Figure $\mathrm{S} 2 \mathrm{C}$ ).

As for the prognostic value of CD204+ TAMs on DFS, the pooled results of three studies ${ }^{17,18,24}$ that elevated CD204+ TAMs density was significantly associated with the DFS $(\mathrm{HR}=1.83,95 \% \mathrm{CI}=1.43-2.36$, fixed effects model, $\mathrm{I}^{2}=0.00 \%, P=0.000$ ) (details in Figure 7).

\section{Impact of CD206+TAMs on survival outcomes}

There was only one study which involved the connection between CD206+ TAMs infiltration and RFS of pancreatic cancer patients. As shown in Table 1, the data presented that CD206+ TAMs correlated with better RFS in pancreatic cancer patients $(\mathrm{HR}=0.24,95 \% \mathrm{CI}=0.12-0.48) .{ }^{23}$

\section{Sensitivity analysis}

We conducted a sensitivity analysis by removing each study to evaluate the effect of individual datasets on the pooled HRs.

In the procedure of investigating the connection between M2-TAMs and OS of pancreatic cancer, we found similar HRs and 95\% CIs except for the situation when we omitted the last included study (details in Figure
$\mathrm{S} 3 \mathrm{~A})$. When we omitted the last included study, ${ }^{11}$ the heterogeneity decreased from $59.2 \%$ to $45.4 \%$. The pooled data changed simultaneously $(\mathrm{HR}=1.83,95 \% \quad \mathrm{CI}$ $=1.63-2.07$, fixed effects model, $P=0.000$ ) (details in Figure 2B). As for the connection between M2-TAMs and DFS of pancreatic cancer, as shown in Figure S3B, we found similar HRs and 95\% CIs, indicating that the result of RFS was relatively stable.

In the procedure of investigating the connection between CD68+ TAMs and OS of pancreatic cancer, we found similar HRs and 95\% CIs except for the situation when we omitted the ninth included study and the last included study ${ }^{11,25}$ (details in Figure S3C). When we omitted the ninth included study and the last included study, ${ }^{11,25}$ the heterogeneity decreased from $82.5 \%$ to $51.2 \%$. As we can see in Figure $4 \mathrm{~B}$, the pooled data also changed. $(\mathrm{HR}=1.51,95 \% \mathrm{CI}$ $=1.10-2.08$, random effects model, $P=0.011$ ).

In the procedure of investigating the connection between CD163+ TAMs and OS of pancreatic cancer, we found similar HRs and 95\% CIs except for the situation when we omitted the last included study (details in Figure S3D). Then, we omitted the last included study, ${ }^{11}$ the heterogeneity decreased from $43.9 \%$ to $24.5 \%$. At the same time, the pooled $\mathrm{HR}$ and $95 \%$ CI changed $(\mathrm{HR}=1.95,95 \%$ $\mathrm{CI}=1.67-2.28$, fixed effects model, $P=0.000$ ) (details in Figure 5B).

In the procedure of investigating the connection between CD204+ TAMs and OS of pancreatic cancer, the results shown in Figure $\mathrm{S} 3 \mathrm{E}$ indicated that the pooled HRs for OS was not substantially changed. As for the connection between CD204+ TAMs and DFS of pancreatic cancer, as shown in Figure S3F, our findings of the pooled HRs were also robust.

\section{Publication bias}

Both Begg's test and Egger's test were performed to estimate the publication bias of the included studies. As shown in Figure S4, Begg's test showed that there was no statistical evidence of publication bias in our metaanalysis. Similarly, Egger's linear regression did not reveal obvious publication bias (detail in Figure S5).

\section{Discussion}

As a kind of malignant tumor, pancreatic cancer is the fourth leading cause of cancer mortality in the world. It is so difficult to diagnose and treat patients with a pancreatic tumor that the five-year survival rate is nearly $5 \%$. The success rate of the radical operation has been improved 
much than ever, but the prognosis of patients who suffer pancreatic cancer has not changed accordingly. Consequently, there is an urgent need to seek for novel therapeutic strategies. Accumulated studies have demonstrated that tumor microenvironment plays particular functional roles in tumor progression, including cancer initiation and promotion, immune suppression, metastasis, establishing a premalignant niche. ${ }^{27,28}$ It is confirmed that tumor microenvironment consists of tumor cells, immune cells, cytokines, and extracellular matrix. ${ }^{29}$ Among them, especially, TAMs occupy a prominent position that orchestrates various factors in the tumor microenvironment, ${ }^{30,31}$ connecting with tumor cells. Therefore, targeting TAMs can be regarded as a novel strategy for the treatment of pancreatic cancers. However, the connection between TAMs and outcome of pancreatic cancer remains contradictory. Our meta-analysis of 13 publications including 1699 patients revealed elevated M2-TAMs infiltration predicted worse OS and DFS. What's more, different from the results in the first, the eighth, and the ninth included studies, ${ }^{10,25,26}$ high-density CD68+ TAMs infiltration connected with worse OS. What's more, our meta-analysis revealed that there was no significance between elevated clinicopathological characteristics and high-density TAMs, neither M2-TAMs nor CD68+ TAMs.

Recently, different subtypes and distribution of TAMs caught attention of many researchers who aim to investigate the roles of TAMs in prognostic prediction comprehensively. In general, TAMs have two polarization subtypes: $\mathrm{M} 1$ and M2. M1 are involved in helper $\mathrm{T}$ cell $(\mathrm{Th}) 1$ response to infection. On the contrary, M2-TAMs contribute to promoting tumor progression. ${ }^{27}$ In most cases, M1 macrophages generally express markers CD80, CD86, human leukocyte antigen (HLA)-DR and inducible nitric oxide synthase (iNOS), while M2 macrophages generally identified by the markers such CD163, CD204, CD206, and Arginase 1. ${ }^{32,33}$ However, CD68, as a common marker, identifies both M1 and M2 TAMs and cannot reflect the TAMs polarization subtypes. So, TAMs labeled by CD68 are also called generalized TAMs. Several studies indicated elevated CD68+ TAMs infiltration did not connect with worse OS. ${ }^{10,25,26}$ The reason might be because of the neutralization of the M1 and M2 prognostic effects. In our meta-analysis, CD68 was used for identification of TAMs in 5 of the 13 included studies and the pooled HRs demonstrated CD68+ TAMs correlated with worse OS in pancreatic cancer patients. At the same time, the heterogeneity is very large, and we need to pay attention when interpreting it. Thus, further studies with larger sample size and more details are still needed to validate our conclusion about CD68+ TAMs. M2-TAMs can be a predictive factor of prognosis in pancreatic cancer patients. CD163, CD204 or CD206 were used for identification of TAMs in all included studies. Although, in our meta-analysis, high CD206+ TAMs density associated with better RFS. However, this result was merely abstracted from one study. ${ }^{23}$ While there was a significant association between M2-TAMs and survival rates of pancreatic cancer patients. The reason that highdensity M2-TAMs associated with poor survival rate (OS and DFS) is the roles of M2-TAMs in tumor progression, including cancer initiation and promotion, immune suppression, metastasis, establishing a premalignant niche. In the aspect of cancer initiation and promotion, M2-TAMs connect inflammation and cancer. TAMs that secreted cytokines IL-23 and IL-17 drive "tumor-elicited inflammation" and promote colorectal cancer progression. ${ }^{34}$ Recent data indicates the increased IL-6 derived from TAMs had an amplifying effect on the inflammation response and further promoted the occurrence and development of hepatocellular carcinoma. ${ }^{35}$ In the aspect of immune suppression, TAMs involved in inhibiting cytotoxic $\mathrm{T}$ lymphocyte (CTL) responses in tumor microenvironments. Evidences indicated the expression of costimulatory molecule PD-L1 in monocytes could be induced by IL-10 derived from TAMs in hepatocellular carcinoma, which can inhibit CTL responses. ${ }^{36}$ In the aspect of cancer initiation and promotion, TAMs promote solid tumor development by providing factors that enhance metastasis and the establishment of a premalignant niche of malignant cells. The epithelialmesenchymal transition (EMT), whose initiation and progression are affected by TAM-derived factors such as transforming growing factor- $\beta_{1}$ (TGF- $\left.\beta_{1}\right)$, plays a fundamental role in tumor progression and metastasis. ${ }^{37}$ Tumor necrosis factor- $\alpha$ (TNF- $\alpha)$, vascular endothelial growth factor (VEGF) and transforming growing factor- $\beta$ (TGF- $\beta$ ) derived from TAMs in cancer tissues, are believed that they can induce macrophages to produce S100A8 and serum amyloid A3 after they reach destination organs. Both of S100A8 and serum amyloid A3 can recruit macrophages and tumor cells to destination organs and promote the formation of metastatic foci. ${ }^{37}$ Taking all into account, targeting TAMs, which are thought to more closely resemble M2-polarized macrophages, may provide us with a novel therapeutic strategy.

To the best of our knowledge, this is the first meaningful meta-analysis shedding light on the prognostic 
value of TAMs in pancreatic cancer. Our study also tells us some important messages. First, although one study suggested high CD206+ TAMs density associated with better relapse-free survival (RFS), it was convinced that the high-density M2-TAMs was associated with poor results in pancreatic cancer patients. Therefore, we can assume that M2-TAMs may provide us with a potential therapeutic target. Elevated density CD68+ TAMs could also predict the prognosis of pancreatic cancer patients. Due to the significant heterogeneity, the prognostic role of CD68+ TAMs remains controversial. Second, it concludes that there was a significant connection between elevated M2TAMs density and OS in pancreatic cancer patients, regardless of the $\mathrm{CD} 163+$ and $\mathrm{CD} 204+$ identified in whole sample area (or not specified), tumor stroma or tumor cell. Besides, M2-TAMs correlated with worse OS in pancreatic cancer patients both in the non-Asian country and the Asian country. Finally, based on the function of TAMs, overall survival of all patients with elevated density TAMs seem to be worse clinicopathological characteristics than those with low-density TAMs, albeit not significantly. In our meta-analysis, the pooled analysis showed that elevated TAMs infiltration had nothing to do with LN metastasis, tumor stage, histological grade, sex, or tumor location. Our study only showed four clinicopathologic factors. There are still other clinicopathologic factors, such as tumor size, venous invasion, margin status, and so on. Kurahara reported the density of CD204+ TAMs in tumors with venous invasion were significantly higher than those without this factor. ${ }^{10}$ Yoshikawa also reported there was a significant connection between cases with portal vein invasion and high central CD204 expression. $^{22}$ As a result, we can guess TAMs have an impact on the prognosis by other ways, like venous invasion. Moreover, only 4 of the 13 included studies have presented relevant data of the association between clinicopathological parameters and TAMs. Therefore, further studies with larger sample size and more detailed information are still needed to validate our conclusion.

However, there are also some limitations existing in our study. First, there was significant heterogeneity among the analysis of M2-TAMs on OS, CD68+ TAMs on OS, and CD204+ TAMs on OS. But it is a common phenomenon that heterogeneity among the studies existed when we conduct a meta-analysis of observational studies. According to the results of sensitivity analysis, the study from Knudsen and his team is the source of statistical heterogeneity in the meta-analysis. In our meta-analysis, the differences in the quality of studies, the country, the number of patients, sample size, cut-off value, tissue distribution and the antibodies and dilution, which was applied to detect the TAMs density, might be the sources of heterogeneity. After we conducted all subgroup analysis, we found that, among the above factors, tissue distribution might be the most critical factor. The reason might be that there was no explicit boundary between three kinds of tissue distribution. This may lead to bias into the evaluation of tumor-associated macrophage infiltration when different searchers investigated them. Moreover, the included studies selected different kinds of antibodies or dilution when the authors detected the density and distribution of TAMs. Third, it has not reached consensus on cut-off value, which was used to identify the density of the TAMs. Besides, studies included in our meta-analysis were limited in the English language. Considering these limitations, large-scale prospective studies with high quality are still needed to validate the conclusion from our result.

In summary, our meta-analysis demonstrated that the density of TAMs has an impact on the survival rate of patients with pancreatic cancer. Our study demonstrated that the elevated density of M2-TAMs predicted poor survival in pancreatic cancer patients, specially CD163+ TAMs and CD204+ TAMs. Elevated density CD68+ TAMs could also predict the prognosis of pancreatic cancer patients. Moreover, further studies and multiple centers clinical trials are required to confirm the result of our study.

\section{Acknowledgments}

This study was supported by grants from the National Science Foundation of Guangdong Province, People's Republic of China (No 2014A030310073, 2017A030313530), Guangdong Provincial Science and Technology Plan projects (No 2009B080701021 and 2010B080701021), Guangdong Province Public Interest Research and Capacity - Building Projects, People's Republic of China (No 2014A020212448), National Natural Science Foundation of China (Grant No. 81701560) and Guangzhou Science and Technology Plan of Scientific Research Projects, People's Republic of China (No 201510010286).

\section{Disclosure}

The authors report no conflicts of interest in this work. 


\section{References}

1. Raraty MGT, Magee CJ, Ghaneh P, Neoptolemos JP. New techniques and agents in the adjuvant therapy of pancreatic cancer. Acta Oncol. 2002;41:582-595. doi:10.1080/028418602321028184

2. Chen W, Zheng R, Baade PD, et al. Cancer statistics in China, 2015. CA Cancer J Clin. 2016;66:115-132. doi:10.3322/caac.21338

3. Teng F, Tian WY, Wang YM, et al. Cancer-associated fibroblasts promote the progression of endometrial cancer via the SDF-1/CXCR4 axis. J Hematol Oncol. 2016;9:8. doi:10.1186/s13045-015-0231-4

4. Genard G, Lucas S, Michiels C. Reprogramming of tumor-associated macrophages with anticancer therapies: radiotherapy versus chemoand immunotherapies. Front Immunol. 2017;8. doi:10.3389/ fimmu. 2017.00828

5. Ohno S, Inagawa H, Dhar DK, et al. Role of tumor-associated macrophages (TAM) in advanced gastric carcinoma: the impact on FasL-mediated counterattack. Anticancer Res. 2005;25:463-470.

6. Yuan X, Zhang J, Li D, et al. Prognostic significance of tumor-associated macrophages in ovarian cancer: a meta-analysis. Gynecol Oncol. 2017;147:181-187. doi:10.1016/j.ygyno.2017.07. 007

7. Dai F, Liu L, Che G, et al. The number and microlocalization of tumor-associated immune cells are associated with patient's survival time in non-small cell lung cancer. BMC Cancer. 2010;10:220. doi:10.1186/1471-2407-10-663

8. Zhou Q, Peng R, Wu X, et al. The density of macrophages in the invasive front is inversely correlated to liver metastasis in colon cancer. $J$ Transl Med. 2010;8:13. doi:10.1186/1479-5876-8-72

9. Ino Y, Yamazaki-Itoh R, Shimada K, et al. Immune cell infiltration as an indicator of the immune microenvironment of pancreatic cancer. Brit J Cancer. 2013;108:914-923. doi:10.1038/bjc.2013.32

10. Kurahara H, Shinchi H, Mataki Y, et al. Significance of M2-polarized tumor-associated macrophage in pancreatic cancer. J Surg Res. 2011;167:e211-e219. doi:10.1016/j.jss.2009.05.026

11. Knudsen ES, Vail P, Balaji U, et al. Stratification of pancreatic ductal adenocarcinoma: combinatorial genetic, stromal, and immunologic markers. Clin Cancer Res. 2017;23:4429-4440. doi:10.1158/10780432.CCR-17-0162

12. Stang A. Critical evaluation of the newcastle-ottawa scale for the assessment of the quality of nonrandomized studies in meta-analyses. Eur J Epidemiol. 2010;25:603-605. doi:10.1007/ s10654-010-9491-z

13. Parmar MKB, Torri V, Stewart L. Extracting summary statistics to perform meta-analyses of the published literature for survival endpoints. Stat Med. 2004;23:2815-2834.

14. Tierney JF, Stewart LA, Ghersi D, Burdett S, Sydes MR. Practical methods for incorporating summary time-to-event data into meta-analysis. Trials. 2007;8:16. doi:10.1186/1745-6215-8-16

15. Higgins JPT, Thompson SG, Deeks JJ, Altman DG. Measuring inconsistency in meta-analyses. BMJ. 2003;327:557-560. doi:10.1136/bmj.327.7414.557

16. Begg CB, Mazumdar M. Operating characteristics of a rank correlation test for publication bias. Biometrics. 1994;50(4):1088-1101. Published by: International Biometric Society Stable. Available from: http://www. jstor.org/stable/2533446. Accessed March 20, 2013.

17. Hou Y, Chao Y, Tung H, Wang H, Shan Y. Coexpression of CD44-positive/CD133-positive cancer stem cells and CD204-positive tumor-associated macrophages is a predictor of survival in pancreatic ductal adenocarcinoma. Cancer-Am Cancer Soc. 2014;120:2766-2777.

18. Takakura K, Ito Z, Suka M, et al. Comprehensive assessment of the prognosis of pancreatic cancer: peripheral blood neutrophil-lymphocyte ratio and immunohistochemical analyses of the tumour site. Scand $J$ Gastroenterol. 2016;51:610-617. doi:10.3109/00365521.2015.1121515
19. Chen SJ, Zhang QB, Zeng LJ, et al. Distribution and clinical significance of TAMs in pancreatic ductal adenocarcinoma: a retrospective analysis in China. Curr Oncol. 2015;22:11-19. doi:10.3747/co.22.2150

20. Liu Q, Niu Z, Li Y, et al. Immunoglobulin G4 (IgG4)-positive plasma cell infiltration is associated with the clinicopathologic traits and prognosis of pancreatic cancer after curative resection. Cancer Immunol Immunother. 2016;65:931-940. doi:10.1007/s00262-016-1853-2

21. Hutcheson J, Balaji U, Porembka MR, et al. Immunologic and metabolic features of pancreatic ductal adenocarcinoma define prognostic subtypes of disease. Clin Cancer Res. 2016;22:3606-3617. doi:10.1158/1078-0432.CCR-15-1883

22. Yoshikawa $\mathrm{K}$, Mitsunaga $\mathrm{S}$, Kinoshita $\mathrm{T}$, et al. Impact of tumor-associated macrophages on invasive ductal carcinoma of the pancreas head. Cancer Sci. 2012;103:2012-2020. doi:10.1111/j.13497006.2012.02411.x

23. Wang WQ, Liu L, Xu HX, et al. Infiltrating immune cells and gene mutations in pancreatic ductal adenocarcinoma. $\mathrm{Br} J$ Surg. 2016;103:1189-1199. doi:10.1002/bjs.10187

24. Sugimoto M, Mitsunaga S, Yoshikawa K, et al. Prognostic impact of M2 macrophages at neural invasion in patients with invasive ductal carcinoma of the pancreas. Eur J Cancer. 2014;50:1900-1908. doi:10.1016/j.ejca.2014.04.010

25. Diana A, Wang LM, D'Costa Z, et al. Prognostic role and correlation of CA9, CD31, CD68 and CD20 with the desmoplastic stroma in pancreatic ductal adenocarcinoma. Oncotarget. 2016;7:72819.

26. Hu H, Hang J, Han T, Zhuo M, Jiao F, Wang L. The M2 phenotype of tumor-associated macrophages in the stroma confers a poor prognosis in pancreatic cancer. Tumor Biology. 2016;37:8657-8664. doi:10.1007/s13277-015-4741-z

27. Grivennikov SI, Greten FR, Karin M. Immunity, inflammation, and cancer. Cell. 2010;140:883-899. doi:10.1016/j.cell.2010.01.025

28. Yang L, Zhang Y. Tumor-associated macrophages: from basic research to clinical application. J Hematol Oncol. 2017;10:12-58. doi:10.1186/s13045-016-0381-z

29. Hanchen L, Xueli F, Jeanmarie H. Tumor microenvironment: the role of the tumor stroma in cancer. $J$ Cell Biochem. 2010;101:805-815.

30. Quail DF, Joyce JA. Microenvironmental regulation of tumor progression and metastasis. Nat Med. 2013;19:1423-1437. doi:10.1038/ nm.3394

31. Chen Y, Zhang S, Wang Q, Zhang X. Tumor-recruited M2 macrophages promote gastric and breast cancer metastasis via M2 macrophage-secreted CHI3L1 protein. $J$ Hematol Oncol. 2017;10:36. doi:10.1186/s13045-017-0408-0

32. Martinez FO, Helming L, Gordon S. Alternative activation of macrophages: an immunologic functional perspective. Annu Rev Immunol. 2009;27:451. doi:10.1146/annurev.immunol.021908.132532

33. Takeya M, Komohara Y. Role of tumor-associated macrophages in human malignancies: friend or foe? Pathol Int. 2016;66:491-505. doi:10.1111/pin. 12440

34. Grivennikov SI, Wang K, Mucida D, et al. Adenoma-linked barrier defects and microbial products drive IL-23/IL-17-mediated tumour growth. Nature. 2012;491:254-258. doi:10.1038/nature11465

35. Kong L, Zhou Y, Bu H, Lv T, Shi Y, Yang J. Deletion of interleukin-6 in monocytes/macrophages suppresses the initiation of hepatocellular carcinoma in mice. J Exp Clin Cancer Res. 2016;35:131. doi:10.1186/s13046-016-0444-6

36. Kuang D, Zhao Q, Peng C, et al. Activated monocytes in peritumoral stroma of hepatocellular carcinoma foster immune privilege and disease progression through PD-L1. J Exp Med. 2009;206:1327-1337. doi:10.1084/jem.20082173

37. Deng YR, Liu WB, Lian ZX, Li X, Hou X. Sorafenib inhibits macrophage-mediated epithelial-mesenchymal transition in hepatocellular carcinoma. Oncotarget. 2016;7:38292-38305. doi:10.18632/ oncotarget.9438 


\section{Supplementary Materials}
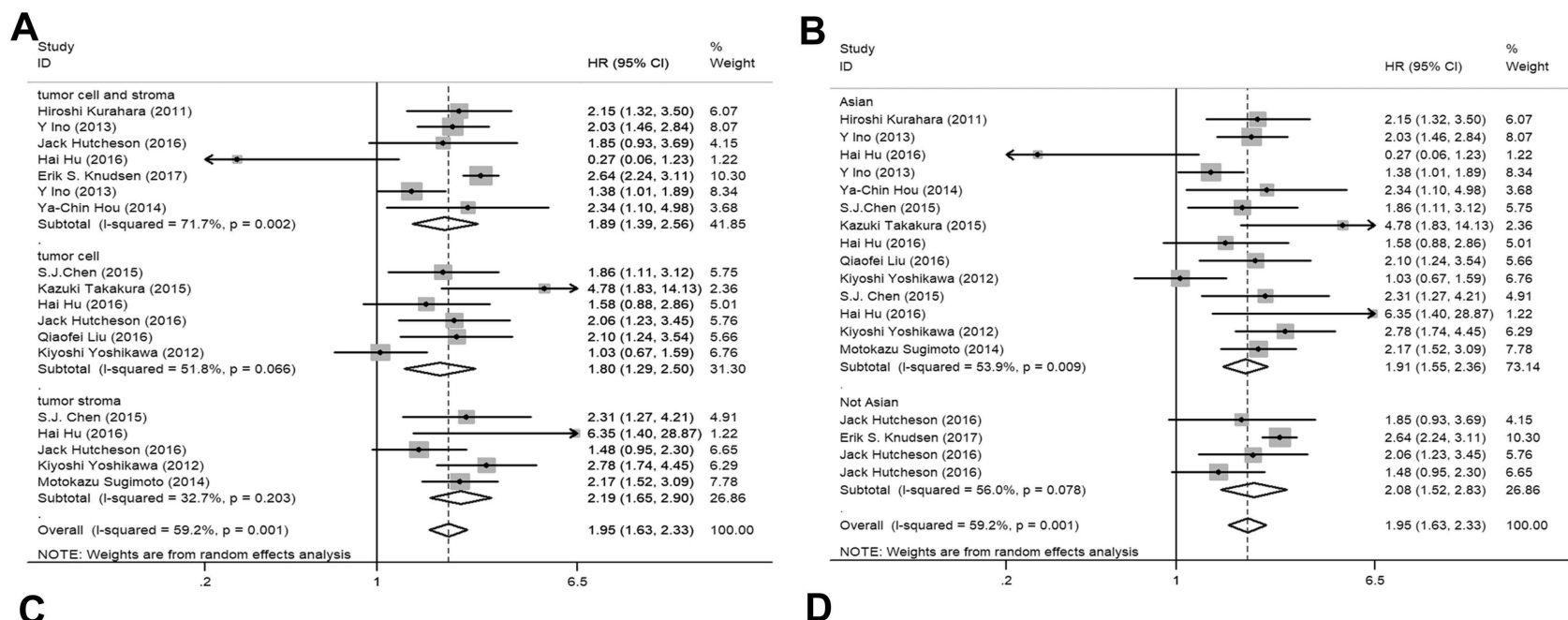

C

D
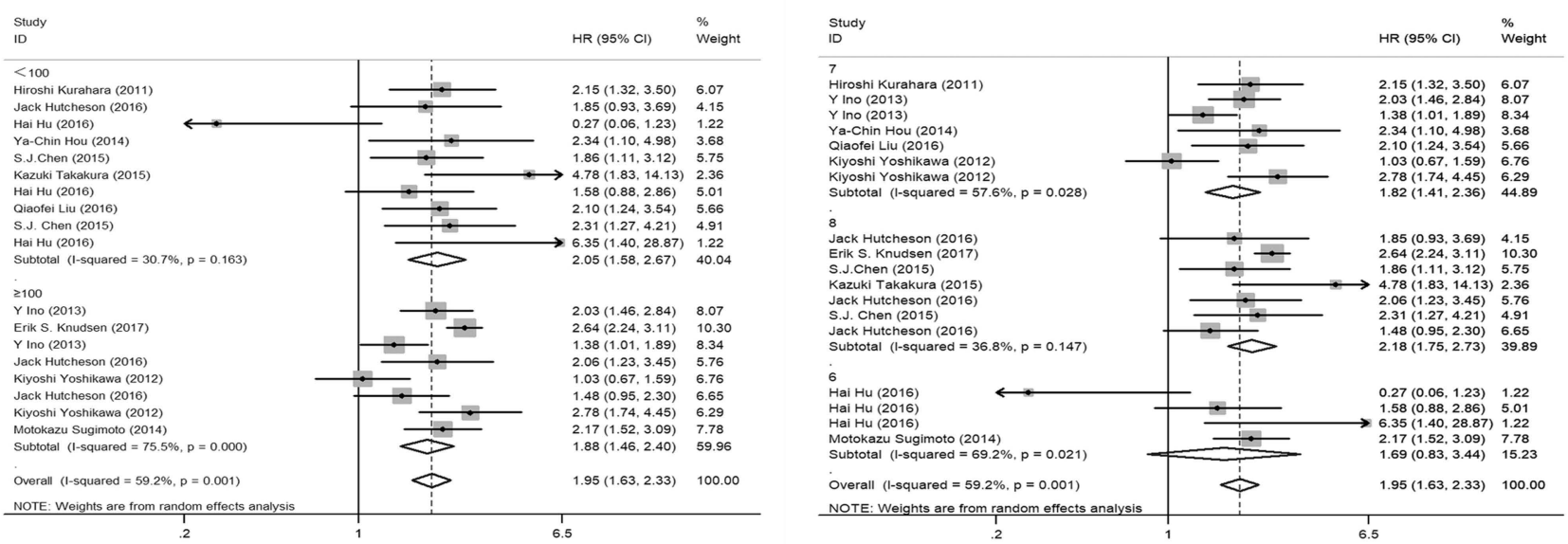

Figure SI Forest plot of hazard ratio (HR) for the subgroup analyses of M2-TAMS: the tissue distribution of paper (A), the country of paper (B), the number of patients of the paper source (C), the quality of the paper (D)
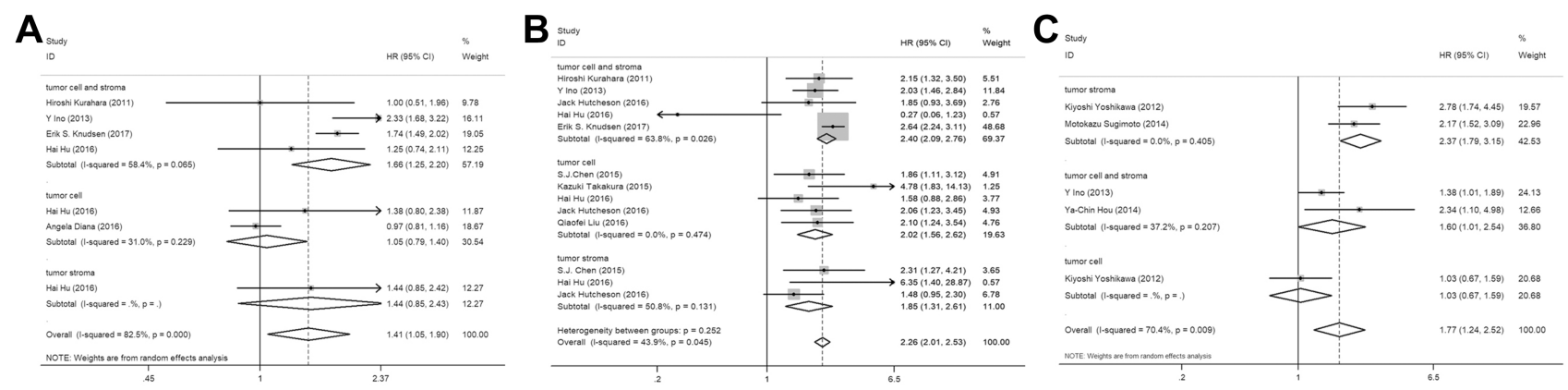

Figure S2 Forest plot of hazard ratio (HR) for the subgroup analyses of the tissue distribution of paper: CD68+ TAMS on OS (A); CDI63+ TAMS on OS (B); CD204+ TAMS on OS (C). 

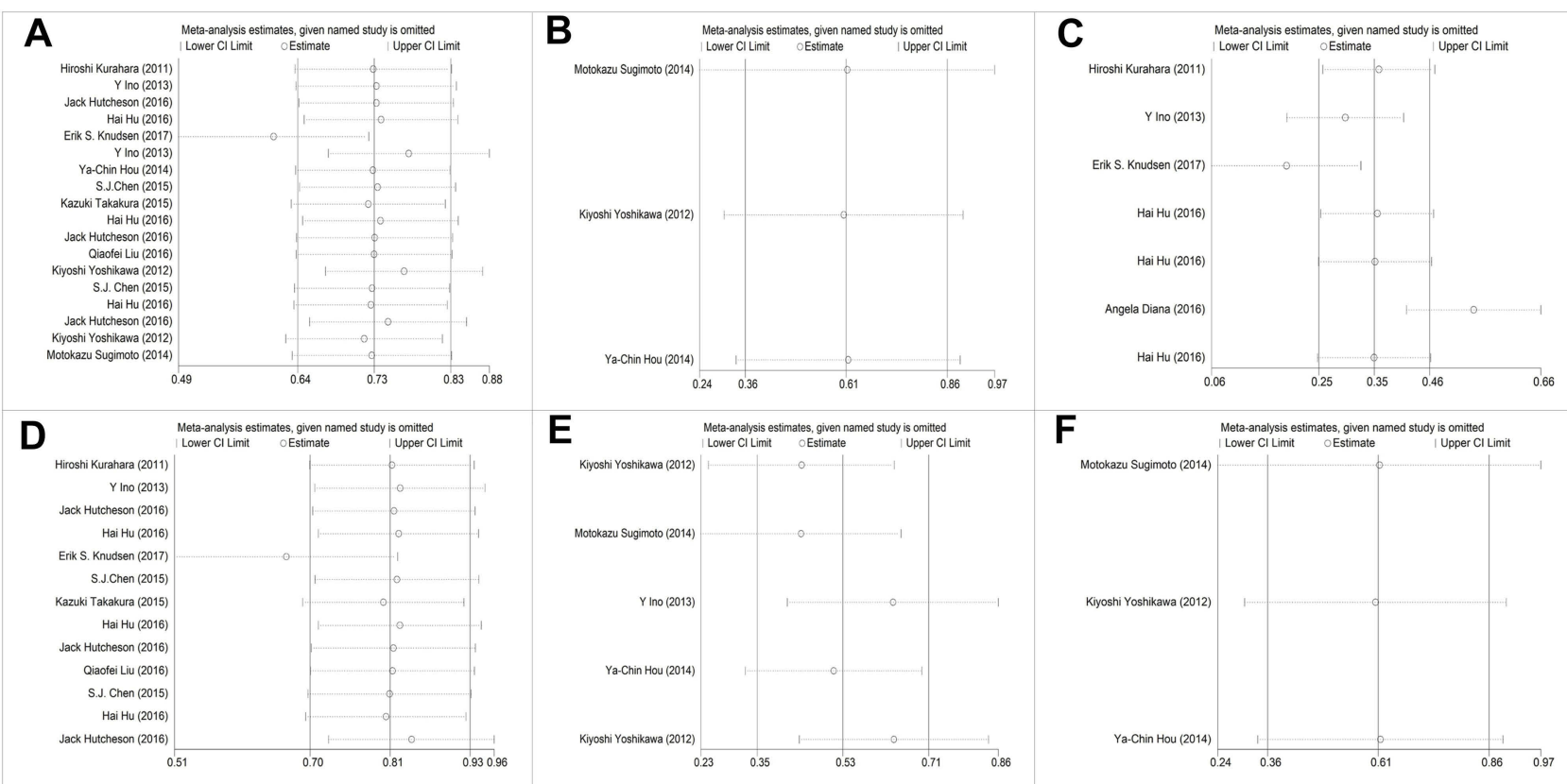

Figure S3 Sensitivity analysis was performed in present studies. (A) M2-TAMS on OS; (B) M2-TAMS on DFS; (C) CD68+ TAMS on OS; (D) CDI63+ TAMS on OS; (E) CD204+ TAMS on OS; (F) CD204+ TAMS on DFS.
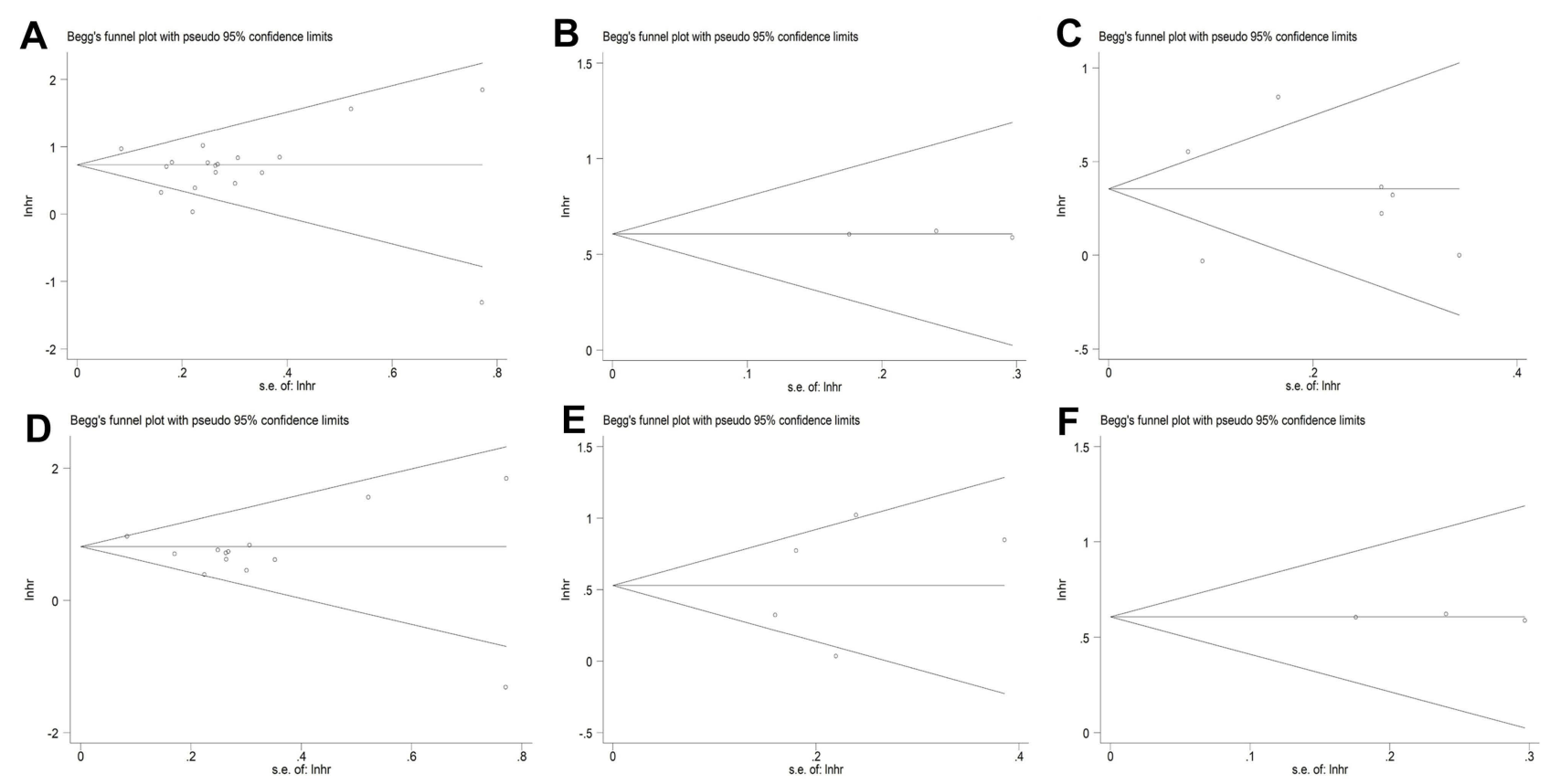

Figure $\mathbf{5} 4$ Begg's test for the assessment of publication bias in the present study, (A) Begg's test of M2-TAMS on OS; (B) Begg's test of M2-TAMS on DFS; (C) Begg's test of CD68+ TAMS on OS; (D) Begg's of CDI63+ TAMS on OS; (E) Begg's test of CD204+ TAMS on OS (F) Begg's test of CD204+ TAMS on DFS. 

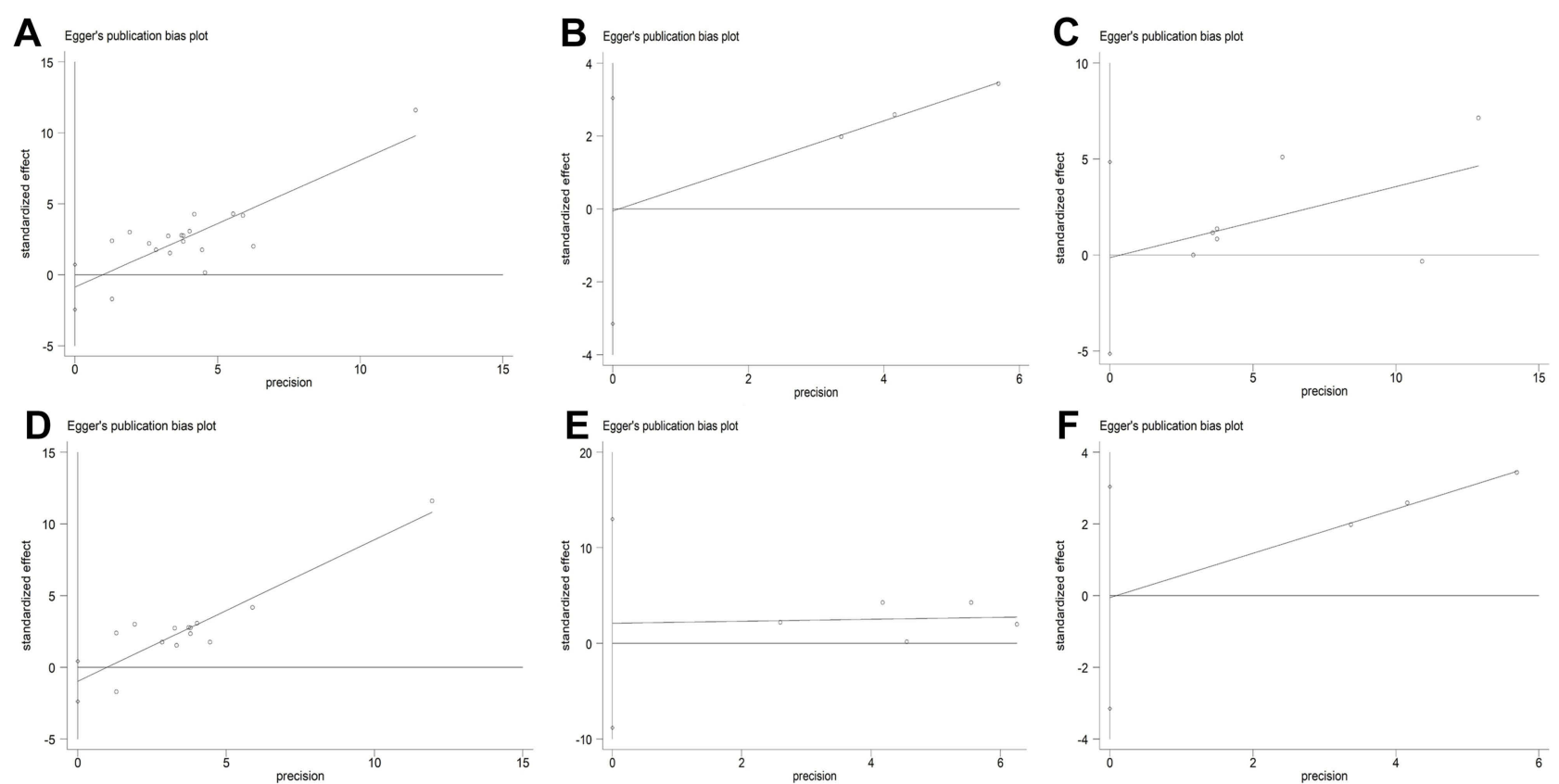

Figure S5 Egger's test for the assessment of publication bias in the present study, (A) Egger's test of M2-TAMS on OS; (B) Egger's test of M2-TAMS on DFS; (C) Egger's test of CD68+ TAMS on OS; (D) Egger's test of CDI63+ TAMS on OS; (E) Egger's test of CD204+ TAMS on OS; (F) Egger's test of CD204+ TAMS on DFS.

\section{Supplementary text 1}

The full electronic strategy of databases

The full electronic strategy for PubMed

The following search terms were variously combined: "pancreatic cancer", "pancreatic carcinoma", "pancreatic duct adenocarcinoma", "pancreatic ductal adenocarcinoma", "macrophage", "tumor-associated-macrophages", "tumorinfiltrating-macrophages", "tumor infiltrating macrophages" and "TAMs". We search the studies which were published before November 2018.

Search formula of PubMed:(\#pancreatic cancer OR \#pancreatic carcinoma OR \#pancreatic duct adenocarcinoma OR \#pancreatic ductal carcinoma) AND (\#macrophage OR \#tumor-associated-macrophages OR \#tumor associated macrophages OR \#TAMs OR \#tumor infiltrating macrophages OR \#tumor-infiltrating-macrophages)

\section{Publish your work in this journal}

Cancer Management and Research is an international, peer-reviewed open access journal focusing on cancer research and the optimal use of preventative and integrated treatment interventions to achieve improved outcomes, enhanced survival and quality of life for the cancer patient.
The manuscript management system is completely online and includes a very quick and fair peer-review system, which is all easy to use. Visit http://www.dovepress.com/testimonials.php to read real quotes from published authors. 